\title{
Modelling The Effect of Cell Shedding on Avascular Tumour Growth
}

\author{
J. P. WARD* and J. R. KING \\ Department of Theoretical Mechanics, University of Nottingham, Nottingham, NG7 2RD, UK
}

(Received 29 January 1998; in final form 28 July 1999)

\begin{abstract}
Earlier mathematical models of the authors which describe avascular tumour growth are extended to incorporate the process of cell shedding, a feature known to affect the growth of multicell spheroids. A continuum of live cells is assumed within which, depending on the concentration of a generic nutrient, movement (described by a velocity field) occurs due to volume changes caused by cell birth and death. The necrotic material is assumed to contain a mixture of basic cellular material (assumed necessary for creating new cells) and a non-utilisable material which may inhibit mitosis. The rate of cell shedding is taken to be proportional to the mitotic rate, with constant of proportionality $\Theta$. Numerical solutions of the resulting system of partial differential equations indicate that, depending on $\Theta$ and the initial conditions, the solution may either tend to the trivial state in finite time (by which we mean complete death of the tumour), or to one of two non-trivial states, namely a steady-state (indicating growth saturation) or a travelling wave (indicating continual linear growth). These long time outcomes are explored by deriving the travelling wave and steady-state limits of the model. Numerical solutions demonstrate that there are two branches of solutions, which we have termed the 'Major' and 'Minor' branches, consisting of both travelling waves and steady-states. The behaviour of the solutions along each branch is discussed, with those of the Major branch expected to be stable. Beyond some critical $\Theta$, where the Major and Minor branches merge, the spheroid ultimately vanishes whatever the initial tumour size due to the effects of cell shedding being too strong for it to survive. The regions of existence of the two long time outcomes are investigated in parameter space, cell shedding being shown to expand significantly the parameter ranges within which growth saturation occurs.
\end{abstract}

Keywords: Tumour growth; mathematical modelling; cell shedding; numerical solution: asymptotic analysis

\section{INTRODUCTION}

Multicell spheroid cultures have been used extensively as in vitro analogues of tumour growth, mimicking the early, avascular stages of tumour development (Folkman and Hochberg, 1973). The growth of these cultures is governed mainly by the penetration of nutrients by diffusion from the external medium into the tumour spheroid, giving rise to a characteristic 'three-stage' pattern of growth (namely

\footnotetext{
${ }^{*}$ Corresponding Author: E-mail: John.Ward@ nottingham.ac.uk
} 
exponential, linear and growth saturation); see Congar and Ziskin (1983), Inch et al. (1970) and Carlsson (1977). A number of other factors are known to influence spheroid growth, of which the self-production of mitotic inhibitor molecules (chalones) has perhaps been the focus of most study (see for example Freyer et al., 1988; Harel et al., 1984; Iversen, 1991). However, a feature which undoubtedly has a major effect on growth, but has been the subject of relatively little work, is the process of cell shedding. This involves the detachment of cells from the surface of a multicell spheroid, after which they disperse into the growth medium; since this medium is frequently renewed (usually about every 2 days), the result is an irretrievable loss of the shed cells. For tumours in vivo it has been speculated that the cell shedding process relates to cell detachment and metastasis (Weiss, 1978; Landry et al., 1981). However, this is uncertain, it being probable that the neighbouring tissues will prevent the escape of many of these cells.

The shedding of cells has often been noted in experimental reports, but there has been little analysis. In Landry et al. (1981), it was observed that cells are more prone to detachment during mitosis and it was claimed that this is due to the weakening of the cell-cell contacts. Cell detachment during mitosis will therefore be the focus of the modelling in this paper. Weiss (1978) showed that exposure of a spheroid to necrotic products from the core also enhances cell shedding. The rate at which cells are lost depends strongly on the cell line. Landry et al. (1981), in their study of EMT6/Ro mammary tumour cultures, examined spheroids of diameters between $400-900 \mu \mathrm{m}$ and estimated the cell shedding rate to be 218 cells $/ \mathrm{mm}^{2} \mathrm{~h}$, which represents $\mathrm{O}(1 \%)$ of the total cell content of the spheroid being lost each hour. In Sasaki et al. (1984), cells were observed to be shed from HeLa S3 multicell spheroids, of diameter $475 \mu \mathrm{m}$, at rates of about $21 \mathrm{cells} / \mathrm{mm}^{2} \mathrm{~h}$; this cell shedding rate was approximately halved when these cells were cocultured with human fibroblast cells. Kohno et al. (1986) studied two cell lines and found cell shedding rates of about 50 cells $/ \mathrm{mm}^{2} \mathrm{~h}$ and 5 cells $/ \mathrm{mm}^{2} \mathrm{~h}$ for DND $1 \mathrm{~A}$ melanoma and PC-10 squamous multicell spheroids, respectively. They also noted that the cell shedding rate was reduced during the application of several chemotherapeutic drugs. Such matters are not addressed here, but provide scope for future modelling.

The process of cell shedding has been included in few mathematical models of multicell spheroid growth, with Landry et al. (1982) and Casciari et al. (1992) being the only known examples. In Landry et al. (1982) the tumour was modelled as a spheroid with an outer rim in which volume is gained through cell birth and a core in which volume is lost by cell death, cell shedding being modelled as a volume sink proportional to the surface area of the spheroid. The resulting model predicts the initial exponential and linear phases of growth, but not the final saturation phase. Their model achieved saturation when it was coupled with the inhibitor model of Shymko and Glass (1976); however, the cells in the resulting saturated spheroid are fully inhibited, i.e. all cells are quiescent, contradicting the experimental observations of Carlsson (1977), Folkman and Hochberg (1973), Freyer and Sutherland (1986) and Haji-Karim and Carlsson (1978), in which a surface layer of cells continues to undergo mitosis. Casciari et al. (1992) used a similar approach to model the cell shedding process in their model of spheroid growth. Their model, however, is considerably more complex than the Landry model, the role of various molecules involved in the cellular respiratory process being considered in order to predict the increase in $\mathrm{pH}$ in the core; however, their model again failed to predict growth saturation. The main aim of these models was to provide good quantitative agreement for the growth of EMT6/Ro mammary multicell spheroids using known parameter values. In the mathematical model described below we aim to examine the role of the cell shedding strength on both the qualitative and quantitative behaviour, to gain a deeper understanding of its effects on spheroid growth.

In this paper, we build on the series of models discussed in Ward and King (1997, 1999a, 1999b), extending them to account for cell shedding. The modelling approach is to assume a continuum of live cells whose growth is driven by a generic nutrient. In the models of Ward and King (1999a) the 
inclusion of mechanisms of necrotic material loss, namely leakage (via diffusion) and consumption by living cells, enabled growth saturation to be predicted, with the surface region being one of cell proliferation (as required by the experimental observations noted above). The modelling was extended in Ward and King (1999b) to investigate the effects of mitotic inhibitors released at cell death. Although the addition of the inhibitors demonstrated little that was qualitatively new, and it was shown that the presence of mitotic inhibitors cannot cause growth saturation on its own, it was shown that they can have significant quantitative effects even at low concentrations. A key motivation of the current work was to investigate whether cell shedding could provide an alternative mechanism for growth saturation. The inclusion of the cell shedding mechanism leads to modification of the boundary conditions employed in the model of Ward and King (1999b) and it will be shown that cell shedding has significant quantitative effects on growth speed and saturation size and gives rise to the existence of two non-trivial long time solutions for a much wider class of parameter sets. A brief discussion of the model derivation together with details of the cell shedding modelling is given in Section 2. In Section 3, the resulting system of partial differential equations is solved numerically, with the effects of changing the extent of cell shedding investigated. As with the previous models, the long time outcomes consist of travelling wave and steady-state solutions, the travelling wave and steady-state limits being studied using both asymptotic and numerical methods in Section 4 . The bifurcations between travelling waves and steady-states and between the existence and nonexistence of non-trivial solution are also discussed.

\section{FORMULATION}

\subsection{Introduction}

We will omit most of the details of the modelling, since much of it is described in Ward and King (1999b); the addition of the cell shedding mechanism requires only the adjustment of the boundary conditions. In summary, the model of avascular tumour growth is based on the assumption that living cells form a continuum, dividing and dying at rates governed by the local concentration of a generic nutrient (e.g. oxygen and glucose) and by the availability materials such as water, proteins and DNA required for the construction of new cells. At cell death a cell is assumed to dissociate into fixed quantities of basic cellular material and non-utilisable material which may inhibit mitosis. For convenience, the non-utilisable material will be termed an inhibitor for the remainder of the paper. An important modelling assumption is that the living cells, cellular material and the inhibitive material occupy all available space within the tumour, leading to the no void condition

$$
V_{L} n+V_{p} p+V_{h} h=1
$$

where $n, p$ and $h$ are the concentrations and $V_{L}, V_{p}$ and $V_{h}$ are the volumes of a living cell, a molecule of cellular material and an inhibitor molecule, respectively. Volume changes due to cell birth and death and due to the diffusion of cellular and inhibitive material creates movement within the tumour described by a velocity field. The resulting system of equations is given in non-dimensional form in Appendix $\mathrm{A}$, and in the next section the modelling of the cell shedding process is described.

\subsection{Modelling Cell Shedding}

The detachment of cells from the surface, and the consequent volume loss, implies that, in contrast to our earlier models, the growth rate $d S / d t$, where $S(t)$ is the coordinate of the tumour surface, is not equal to the surface velocity. The volume loss rate due to cell shedding is given by $4 \pi S^{2}(v(S, t)-d S(t) / d t)$, where $v$ is the internal spheroid velocity. The volume lost is assumed to consist of living cells and cellular and inhibitive material at quantities proportional to their surface densities; defining $N(t)$ to be the number of cells lost through shedding, the rate at which the surface cells are shed is thus given by

$$
\frac{d N}{d t}=4 \pi S^{2}\left(v(S, t)-\frac{d S}{d t}(t)\right) n(S, t) .
$$


The rate of cell shedding is assumed to be proportional to the number of surface cells, by which we mean the cells that occupy the space between $S$ and $S-2 r_{0}$, where $r_{0}$ is the radius of cell; the number of cells susceptible to shedding is therefore given by $n(S, t) 4 \pi\left(S^{3}-\left(S-2 r_{0}\right)^{3}\right) / 3$. We can thus write the cell shedding rate as

$$
\frac{d N}{d t}=8 \pi r_{0} S^{2} F\left(S / r_{0}\right) K(c, n, p, h) n(S, t),
$$

where $c$ is the nutrient concentration,

$$
F(\mathcal{S})=1-\frac{2}{\mathcal{S}}+\frac{4}{3 \mathcal{S}^{2}}
$$

and $K(c, n, p, h)$ is some function describing the effects of the microenvironment on the cell shedding rate. Eliminating $d N / d t$ between Equations (2) and (3) results in a differential equation for the moving boundary $S(t)$, namely

$$
\frac{d S}{d t}=v(S, t)-2 r_{0} K(c, n, p, h) F\left(S / r_{0}\right)
$$

We note the model of Ward and King (1999b) can be derived simply by setting $K \equiv 0$, reducing (5) to $d S / d t=v(S, t)$. Our continuum modelling is only really valid for $S \gg r_{0}$, so it might seem reasonable to set $F(\mathcal{S})=1$; however, while this condition is typically satisfied for almost all of the growth, we shall start from an initial state with $S(0)=r_{0}$, and the full form (4) is then needed if the cell shedding rate is not always to be too strong for smaller spheroids; the form (4) is thus adopted as the most appropriate way to describe all stages of growth. Nevertheless, as before (Ward and King, 1997) results of the continuum model on very early timescales, for which $S / r_{0}=O(1)$, should be treated with caution, as the continuum assumption on living cells only holds if there are a large number of cells.

In view of the observations of Landry et al. (1981) that cells are more prone to detach during mitosis, we assume $K(c, n, p, h)$ to be proportional to the mitotic rate, $k_{m}$, at the spheroid surface, so that

$$
K(c, n, p, h)=\Theta k_{m}(c(S, t), p(S, t), h(S, t)) .
$$

Here, the dimensionless constant $\Theta$ is the proportion of the surface layer cells that are undergoing mitosis which detach. As the spheroid develops, so that $k_{m}(c(S, t) ; p(S, t), h(S, t))$ becomes approximately constant, this choice for function $K$ approaches the cell shedding term used by Landry et al. (1982) of cell shedding rate being proportional to the surface area. We note also that defining $K(c, n, p, h)$ in this way means that the model for cell shedding contains only one more parameter than the model of Ward and King (1999b), namely $\Theta$, values for which can be obtained from the literature.

Experimental data is usually expressed in terms of the rate of cell shedding per unit area $\left(R_{C S}\right)$, which is simply $(d N / d t) / 4 \pi S^{2}$, so from (3) we have

$$
R_{C S}=2 \Theta r_{0} \tilde{k}_{m} n(S, t) F\left(S / r_{0}\right),
$$

where $\tilde{k}_{m}$ is the mitotic rate at the spheroid surface. Equation (6) can be used to determine suitable values for $\Theta$ from existing data. For example, using the cell shedding rate of $218 \mathrm{cells} / \mathrm{mm}^{2} \mathrm{~h}$ given in Landry et al. (1981) and the data listed in the Appendix of Ward and King (1997), whereby $n(S, t) \approx 1 / V_{L}=$ $10^{9} \mathrm{~cm}^{-3}, r_{0} \approx 6 \times 10^{-4} \mathrm{~cm}, \tilde{k}_{m} \approx A \approx 10^{-5} \mathrm{~s}^{-1}(A$ being the maximum mitotic rate) and $F\left(S / r_{0}\right) \approx 1$ (which holds for $S \gg r_{0}$ ) we have $\Theta \approx 0.5$. $\Theta$ will be varied in this paper to obtain an understanding of its effects on growth.

The difference between growth rate and surface velocity in (5) also requires a modification of the Robin boundary conditions used in Ward and King (1999b) to describe the cellular and inhibitive material flux at the spheroid surface. The cell shedding modification gives at $r=S(t)$

$$
\begin{aligned}
& D_{p} \frac{\partial p}{\partial r}=Q_{p}\left(p_{0}-p\right)+\left(v-\frac{d S}{d t}\right) p, \\
& D_{h} \frac{\partial h}{\partial r}=Q_{h}\left(h_{0}-h\right)+\left(v-\frac{d S}{d t}\right) h,
\end{aligned}
$$

where $Q_{p}$ and $Q_{h}$ are the mass transfer coefficients and $p_{0}$ and $h_{0}$ are the external concentrations of the cellular and inhibitive material, respectively.

The full system of dimensionless equations implementing the cell shedding process is given in Appendix A. Before discussing numerical solutions of the model we first describe special cases relating the current model to previous works and then 
introduce classifications of the long time solutions which are of relevance to the later sections.

It was found necessary in some of the simulations described later to allow the spheroid to grow without cell detachment before switching on the cell shedding mechanism, to enable investigation of non-trivial large time outcomes by preventing extinction of the spheroid when it is small in size; as we shall see, an important effect of incorporating cell shedding is to cause spheroids of less than some critical size to cease to be viable. We note that it is possible that cell lines with inherently high cell shedding rates, corresponding to very low cell-cell adhesion, may be unable to form spheroids.

\subsection{Special Cases}

The cell shedding model is a generalisation of the models described in Ward and King (1997, 1999a and 1999b). The following are also special cases and will be discussed later in this paper. We note that all quantities discussed here are dimensionless and described in more detail in Appendix A.

\section{Cell shedding-no inhibitor model}

For this model we set the parameters so that there is no production or external source of the mitotic inhibitor, i.e. we take $\mu=0$ and $h_{0}=0$.

\section{Cell shedding-leakage only model}

Here there is no cellular material consumption or inhibitor production, so that leakage is the only mechanism for necrotic volume loss. This is achieved by setting $p_{c}=0$ and $\lambda=0$, preventing utilisation of the cellular material during mitosis, and $\mu=0$ and $h_{0}=0$, so that there is no source of inhibitor. As with the Leakage only model of Ward and King (1999a) these conditions may lead to either growth saturation or a travelling wave.

\section{Cell shedding-inhibition model}

This is obtained by setting $Q_{p}=Q_{h}=0$ (preventing leakage), $\lambda=p_{c}=0$ (preventing consumption of the cellular material) and $\nu=1$ (preventing volume loss through inhibitor breakdown). It will be shown in Section 4.2 that, except in the case of $\delta=0$ where no inhibitor is produced, only travelling wave solutions or tumour extinction can then result in large time.

\section{No leakage model}

This is derived by setting $Q_{p}=Q_{h}=0$. It will be shown that the tumour dies out for all $\Theta \geq 0$ if $\delta-(1-\nu) \mu>\lambda$.

\subsection{Classification of Long Time Solutions}

For the case $\Theta=0$, it was shown in Ward and King (1999a) that there may be zero, one or two branches of travelling wave and steady-state (i.e. non-trivial) solutions, depending on the parameter values. It will be convenient below to use the terms Regime I and Regime II, introduced in Ward and King (1999b), and defined as follows.

\section{Regime I}

Parameter sets (with $\Theta=0$ ) for which only one branch of non-trivial long time solutions exists. These occur when $a\left(1, p_{0}, h_{0}\right)>0$.

\section{Regime II}

Parameter sets (with $\Theta=0$ ) for which either two or zero branches of non-trivial long time solutions exist. These occur when $a\left(1, p_{0}, h_{0}\right)<0$.

These regimes can be identified by, for example, studying the travelling wave and steady-state limits of Equations (20)-(22) in the limit of $\lambda \rightarrow \infty$; see Ward and King (1999a, 1999b). Defining $n_{0}$ by $a(1,1-$ $\left.n_{0}-h_{0}, h_{0}\right)=0$, it can be shown for $\Theta=0$ that if $n_{0}+p_{0}+h_{0} \geq 1$ then the long time solutions lie in Regime I, otherwise the solutions lie in Regime II; the inequalities above follow because $a(c, p, h)$ is monotonic increasing in $p$. Regime $I$ is relevant for all $\lambda$, but non-trivial Regime II solutions are restricted to a finite range of $\lambda$; which case applies depends on the values of the other parameters. 
For $\Theta>0$ the situation is rather different. As will be apparent from the studies of the long time behaviour in Sections 3 and 4 below, the addition of the cell shedding term leads to the existence of two non-trivial branches of travelling wave and steadystate solutions which, like Regime II solutions, can be characterised by the ultimate size of the spheroid. A typical example is given in Figure 1, where the curves are generated from numerical solutions to the systems derived in Section 4. The two branches of long time solutions are illustrated by the solid and dashed curves in the figure; we expect that the dashed solutions are unstable to radially symmetric perturbations, and this is discussed in the Appendix B for the limit $\Theta \rightarrow 0$ with $S=O\left(\Theta^{1 / 3}\right)$. To the left of the dotted line (which is the travelling wave/steadystate bifurcation on the upper branch), both travelling wave (not shown) and steady-state solutions exist, whilst to the right, both long time solutions are steady-states. To distinguish between these branches, it is convenient to introduce the terms 'Major' and 'Minor' branch, as follows.

\section{Major branch solution}

As $\Theta \rightarrow 0$, these solutions tend smoothly to the non-trivial $\Theta=0$ solution (under Regime I) or the upper branch $\Theta=0$ solution (under Regime II). The long-time growth velocity and saturation size are monotonic decreasing in $\Theta$; at the turning point the Major and Minor branches meet. These solutions are expected to be stable.

\section{Minor branch solution}

As $\Theta \rightarrow 0$, these solutions tend smoothly to the trivial solution (under Regime I) or the lower branch $\Theta=0$ solution (under Regime II); an example of this behaviour will be given in Section 4. It appears that the long time solution on this branch is always a steady-state, with the saturation size mostly increasing in $\Theta$ (until the turning point is reached). There is the possibility of there being additional folds along this branch depending on the form of function $F(\mathcal{S})$ in Equation (4); an example of this behaviour is discussed in Section 4.3. These solutions are expected to be unstable.

These branches meet at some critical value of $\Theta=$ $\Theta_{c}$, with no non-trivial long time solutions existing for $\Theta>\Theta_{c}$ due to the cell shedding rate being too strong, so that the spheroid eventually vanishes.

We note that the curve of saturation size against $\Theta$ illustrated in Figure 1 is qualitatively similar to that in Figure 3 of Byrne and Chaplain (1996), where saturation size is plotted against a term proportional to the surface tension per unit surface area, $\gamma$. However, an increasing $\Theta$ can be thought of as corresponding to reduction in cell-cell adhesion forces, the physical

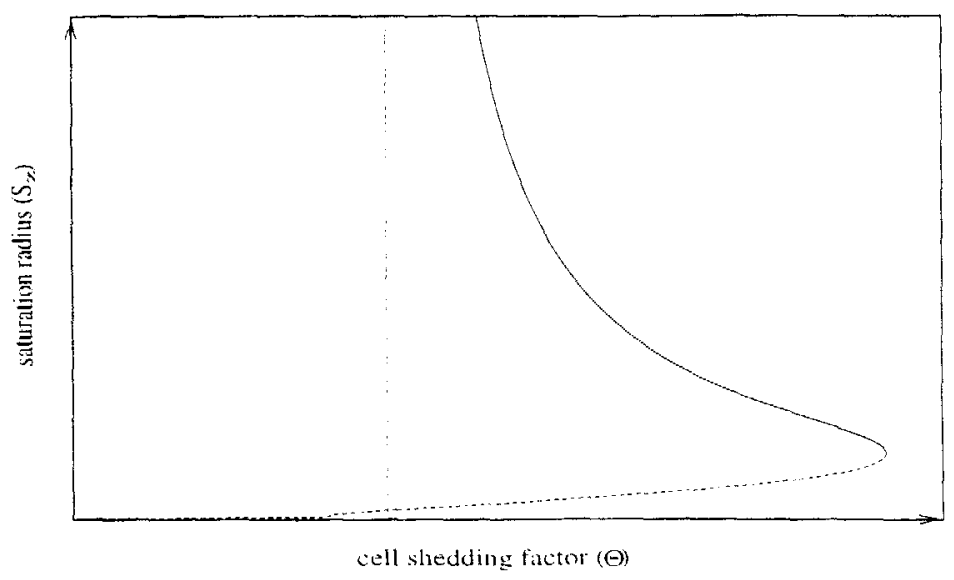

FIGURE 1 Figures showing the effects of the cell shedding factor $\Theta$ on the saturation (steady-state) spheroid radius. The solid and dashed curves respectively illustrate the Major and Minor branch long time solutions, and the dotted curve indicates the travelling wave/steady-state bifurcation. 
mechanisms responsible for the fold are therefore quite different.

\section{NUMERICAL RESULTS}

The numerical procedure used to approximate the system (20)-(23) subject to (26) is essentially the same as that described in Ward and King (1999a). The system is rescaled to fix the moving boundary to unity using $r=S(t) \rho$ and is solved using finite differences in a predictor-corrector type fashion, NAG routines being used on Equations (20), (21) and (22). Full details are given in Ward (1997).

Greater insight into the effects of cell shedding is gained from the study of the long time system, so only a few examples of the transient behaviour are given here. The basic set of parameters used are given by (19) and (20) in Ward and King (1999b), namely

$$
\begin{aligned}
& B / A=0.5, \quad \sigma=0.9, \quad c_{c}=0.1, \quad c_{d}=0.1, \\
& m_{1}=1, \quad m_{2}=1, \quad \beta_{1}=0.01, \quad \beta_{2}=0 \\
& \delta=1, \quad \lambda=1, \quad D_{p}=300, \quad Q_{p}=10 \\
& p_{0}=0.1, \quad p_{c}=0.1, \quad m_{3}=1, \quad \mu=0.1 \\
& \psi=1, \quad \nu=1, \quad P=0.3, \quad D_{h}=300
\end{aligned}
$$

$$
Q_{h}=10, \quad h_{c}=0.1, \quad h_{0}=0,
$$

with $\Theta$ being varied. As described in Ward and King (1999b), these parameters are based on a combination of experimental data and suitable estimates. In particular, there is assumed to be no volume loss at cell death $(\delta=1)$ and the necrotic material consists of $90 \%$ cellular and $10 \%$ inhibitive materials (implied by $\mu=0.1$ ). The diffusive and leakage properties of the cellular and inhibitive material are taken to be equal. The external medium contains some cellular material $\left(p_{0}=0.1\right)$ but no inhibitor $\left(h_{0}=0\right)$. The inhibitive material can at most reduce the mitotic rate by $30 \%$ $(P=0.3)$ and can be completely converted by the living cells to cellular material $(\nu=1)$.

Figure 2 show the effects of $\Theta$ on spheroid growth and on cell population loss rates, using the data given by (7); the curve for $\Theta=0$ in Figure 2 is the same as that shown in Figure 1 in Ward and King (1999b). In these simulations the cell shedding term was switched on at $t=25$. so that the spheroid had reached a sufficient size for growth to continue, where possible, to a non-trivial long time solution. The switching on of the cell shedding term in this manner is probably not unreasonable, as the population reached by $t=25$ reflects the initial population of experimentally grown

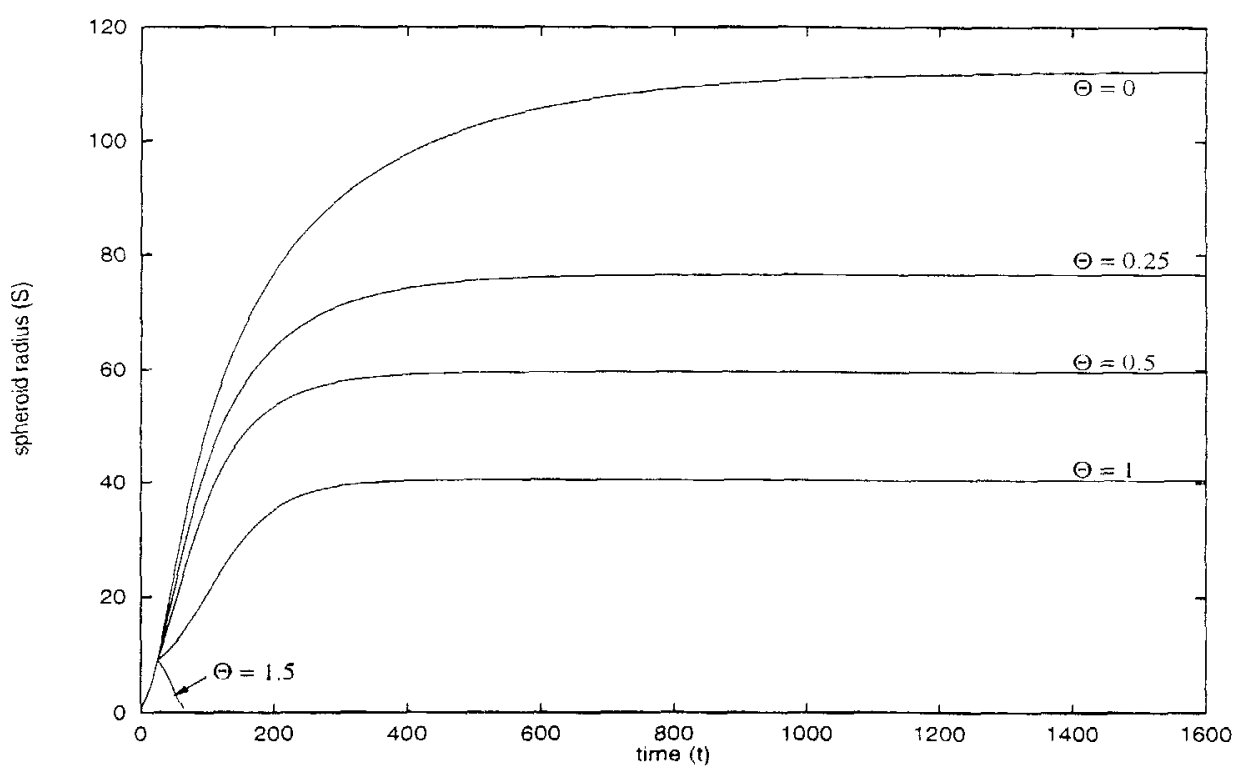

FIGURE 2 Tumour radius against time for various values of the cell shedding parameter $\Theta$. The curves coinicide until $t=25$, when cell shedding is turned on. 
multicell-spheroids which is typically about $10^{4}$ cells. The figure demonstrates that the saturation size is strongly dependent on $\Theta$ and, as expected, increasing $\Theta$ reduces the spheroid saturation size. Beyond a bifurcation value of $\Theta_{c} \approx 1.46$ (see next section) the cell shedding rate becomes so strong that the spheroid eventually vanishes (an example of this being given for $\Theta=1.5$ ). Calculating the rates of cell population loss per unit area $\left(R_{C S}\right)$ using Equation (6), reverting to dimensional quantities (using $A=10^{-5} \mathrm{~s}^{-1}$, $r_{0}=6.2 \times 10^{-4} \mathrm{~cm}$; see the Appendix in Ward and King, 1997), comparison can be made between these results and experimental results from the literature. In this simulation we find at growth saturation that $R_{C S}$ is about $1.0,1.9$ and 3.0 cells $/ \mathrm{cm}^{2}$ s for $\Theta=0.25,0.5$ and 1 , respectively, these being in line with the experimental results of Landry et al. (1981, 6 cells $/ \mathrm{cm}^{2} \mathrm{~s}$ ). Sasaki et al. (1984, $\left.0.6 \mathrm{cells} / \mathrm{cm}^{2} \mathrm{~s}\right)$ and Kohno et al. $\left(1986,1.4\right.$ and 0.13 cells $/ \mathrm{cm}^{2} \mathrm{~s}$ ).

In Figure 3 the spheroid radius is plotted against time for the Cell shedding-leakage only case, using the parameter set (7) but with $D_{p}=800$ and $Q_{p}=$ 100 , so that the curve for $\Theta=0$ is the same as the curve for $D_{p}=800$ in Figure 11 of Ward and King (1999a). For $\Theta>0$, cell shedding was switched on at $t=10$, again to give the spheroid a chance to grow. For $\Theta=0$ and 0.25 growth ultimately becomes linear, whilst for $\Theta=0.5,0.75$ and 1 it saturates, the bifurcation occurring at $\Theta_{c} \approx 0.39$. We note that growth again tends towards solutions on the Major branch in these examples. The decrease of both the linear growth speed and the saturation size on increasing $\Theta$ is to be expected, and this figure demonstrates how the inclusion of cell shedding can induce growth saturation when it would not otherwise occur. It can be observed numerically that the cell loss rate per unit area levels off in all cases, with rates $R_{C S}$ of $2.5,5.1,7.6,10.1 \mathrm{cells} / \mathrm{cm}^{2} \mathrm{~s}$ for $\Theta=0.25,0.5,0.75$, 1. respectively. again comparable with experimental results.

An investigation into the stability of a particular Minor branch solution is illustrated in Figure 4, using the smaller of the two steady-state solutions $\left(S_{\infty}^{m}<S_{\infty}^{M}\right.$, these denoting the saturation sizes on Minor and Major branches, respectively) of the Cell shedding-leakage only model (found by solving the appropriate system from the next section), with parameter values given by the first three rows of (7) and with $\lambda=0.8, D_{p}=Q_{p}=300$ and $\Theta=0.8$. The steady-state was used as the initial condition, except

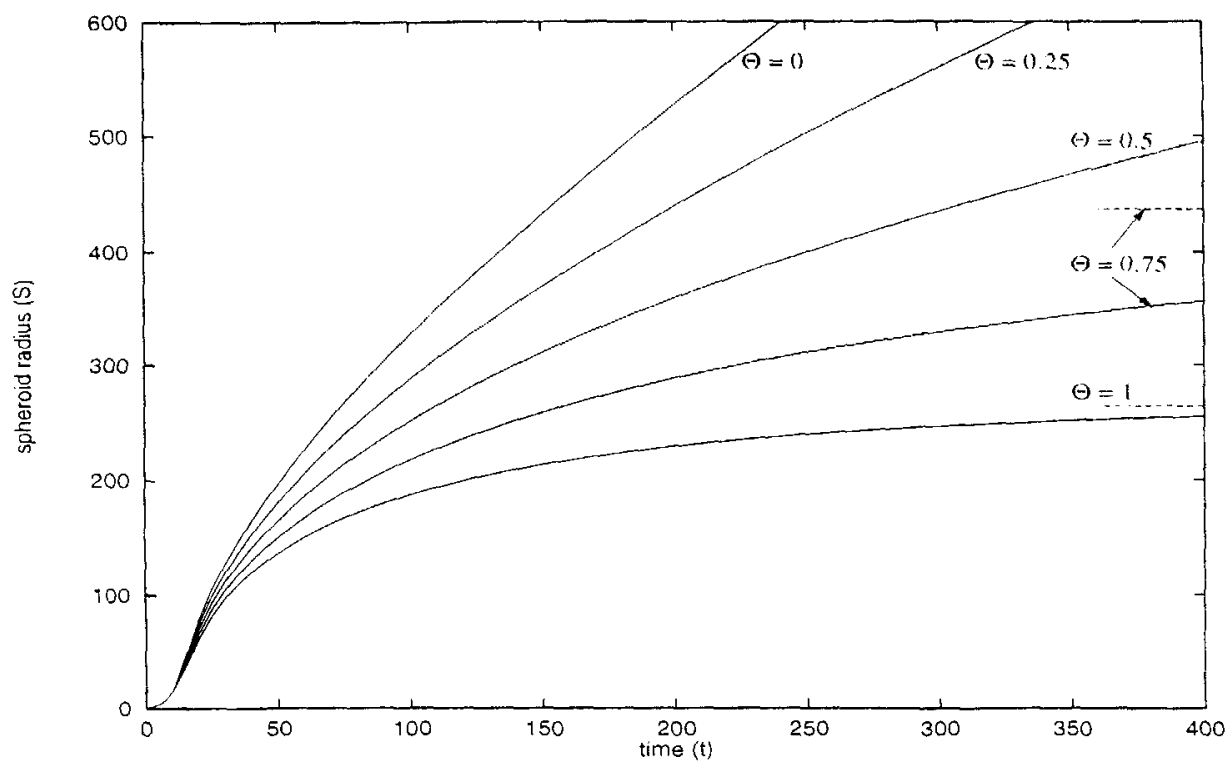

FIGURE 3 Tumour radius against time for various values of the cell shedding parameter $\Theta$, without inhibition or mitotic contraction. The dashed lines indicate the saturation radii, the saturation size for $\Theta=0.5$ being $S_{\infty} \approx 1339$. Growth does not saturate for $\Theta=0$ or $\Theta=0.25$. 


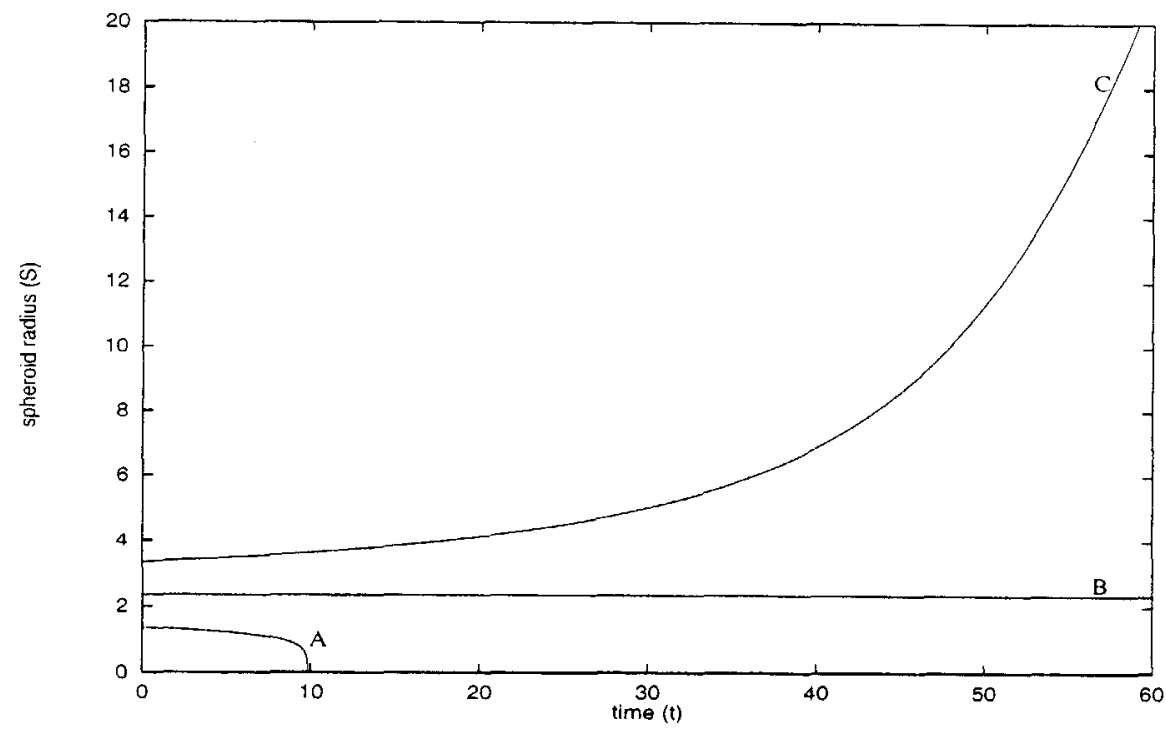

FIGURE 4 Spheroid radius against time for various initial sizes: $S(0) \approx 1.35$ (curve $\mathbf{A}$ ), $S(0) \approx 2.35$ (curve $\mathbf{B}$ ) and $S(0) \approx 3.35$ (curve $\mathbf{C}$ ).

that the initial size was varied and the dependence on $r$ scaled accordingly. The curve labelled $\mathbf{B}$ starts with the actual steady-state solution (with $S_{\infty}^{m}=S(0) \approx$ 2.35), and there is negligible deviation from this initial state. However, perturbing the initial size above or below, as shown by curves $\mathbf{A}$ and $\mathbf{C}$, leads to growth diverging away from the curve $\mathbf{B}$ solution, the smaller spheroid vanishing in finite time and the larger growing towards a Major branch steady-state solution $\left(S_{\infty}^{M} \approx 128.6\right.$ ). We note that this extinction in finite time is in contrast to the decay in infinite time of solutions under a Regime II parameter set without cell shedding (cf. Figure 10 of Ward and King, 1999a). This finite time extinction behaviour is predicted by the analysis in the Appendix B, which demonstrates that Minor branch solutions are unstable in the limit $\Theta \rightarrow 0$; it is expected that solutions on this branch are always unstable.

\section{LONG TIME BEHAVIOUR}

\subsection{Long Time Equations}

As with the model of Ward and King (1999b), the possible long time outcomes of Equations (20)-(22) are travelling wave (with a priori unknown speed
$U$ ) and steady-state (of a priori unknown saturation size $S_{\infty}$ ). The form of the nonlinear diffusion term in (20) and (22) leads to the possibility of steady-state solutions with fully developed ( $n \equiv 0$ for $r<R_{\infty}<$ $S_{\infty}$, for some $R_{\infty}$ ), as well as a partially developed ( $n>0$ for all $r$ ), necrotic cores. The long time systems of equations for each case are listed in Ward and King (1999b) and are omitted for brevity.

\subsection{Existence of Non-trivial Solutions}

In this section we examine the existence of non-trivial solutions for the Cell shedding-inhibitor model and the No leakage model presented in Section 2.3. For the Cell shedding-inhibitor model, it will be demonstrated that, except in a special case, steady-state solutions do not occur. However, unlike our earlier models, steady-state solutions of the No leakage model exist over a range of parameters rather than only along a three-dimensional 'surface' in $(\delta, \lambda, \mu, \nu)$ parameter space; non-trivial solutions exist only in a bounded region in parameter space, defined below.

\subsubsection{Cell shedding-inhibitor model}

Recalling that for the Cell shedding-inhibitor model we have $Q_{p}=Q_{h}=\lambda=p_{c}=0$ and $\nu=1$, the 
travelling wave equations for $n$ and $v$ can be written as

$$
\begin{aligned}
{[(v-U) n]^{\prime}=} & \left(k_{m}-k_{d}\right) n \\
v^{\prime}= & \left(k_{m}-(1-\delta) k_{d}\right) n-D_{p}\left(n^{\prime \prime}+h^{\prime \prime}\right) \\
& +D_{h} h^{\prime \prime},
\end{aligned}
$$

from which we can deduce

$$
v^{\prime}=[(v-U) n]^{\prime}+\delta k_{d} n-D_{p}\left(n^{\prime \prime}+h^{\prime \prime}\right)+D_{h} h^{\prime \prime},
$$

subject to $D_{p}\left(n^{\prime}(0)+h^{\prime}(0)\right)=(v(0)-U)(1-n(0)-$ $h(0))$ and $D_{h}=v(0) h(0)$. On integration this yields $U=\delta \int_{-\infty}^{0} k_{d} n d z$. Positivity of the wave speed implies that travelling wave solutions exist only if $\delta$, the ratio of dead to live cell volumes, is greater than zero. Repeating the analysis on the steady-state equations results in $\delta \int_{0}^{S_{x}} r^{2} k_{d} n d r=0$, implying that non-trivial solutions exist only if $\delta=0$. In summary we have

$\delta>0$ : Only travelling wave solutions exist.

$\delta=0$ : Only steady-state solutions exist; these are non-unique, being dependent on the initial conditions (see Section 5.1 of Ward and King, 1999b, for full details).

Thus the material leakage and/or consumption mechanisms are essential for growth saturation to be predicted across a general parameter range.

\subsubsection{No leakage model}

Recalling that for this limit of the model we have $Q_{p}=Q_{h}=0$, so using (14) together with

$$
\begin{aligned}
v^{\prime}= & {\left[(1-\lambda) k_{m}-(1-\delta) k_{d}-(1-\nu)\right.} \\
& \times \psi h] n-D_{p}\left(n^{\prime \prime}+h^{\prime \prime}\right)+D_{h} h^{\prime \prime} \\
{[(v-U) h]^{\prime}=} & D_{h} h^{n}+\left(\mu k_{d}-\psi h\right) n
\end{aligned}
$$

we may deduce for the case $\psi>0$

$$
\begin{aligned}
& U(1-(1-\nu) h(-\infty))+\lambda(v(0)-U) n(0) \\
& \quad=(\delta-\lambda-(1-\nu) \mu) \int_{-\infty}^{0} k_{d} n d z
\end{aligned}
$$

using (8)-(10) with $Q_{p}=Q_{h}=0$. In the case of $\psi=0$, that is the inhibitor is not broken down, the results to follow are the same as for $\nu=1$. With $v(0)>U$ and $(1-\nu) h(-\infty) \leq 1$ the left-hand side is positive, so positivity of the right-hand side implies that

$$
\delta-(1-\nu) \mu>\lambda
$$

is a necessary condition for the existence of travelling wave solutions in this no leakage case. Equation (15) states that the total amount of cellular material that can be produced through cell death $(\delta-(1-\nu) \mu)$ needs to exceed the amount consumed at cell birth $(\lambda)$. A condition for a steady-state solution can be derived in a similar way, resulting in expression

$$
\lambda v\left(S_{\infty}\right) n\left(S_{\infty}\right)=\frac{(\delta-\lambda-(1-\nu) \mu)}{S_{\infty}^{2}} \int_{0}^{S_{\infty}} r^{2} k_{d} n d r
$$

where the inclusion of cell shedding in the model implies that $v(0)>0$ and $\lambda>0$, so that the left-hand side is positive, implying that (15) must again hold for a non-trivial steady-state to exist. In the case of $\lambda=0$, i.e. zero cellular material volume, the left hand side of (16) is zero, implying that for steady-state solutions we must have $\delta=(1-\nu) \mu$; since $\delta \geq \mu \geq(1-\nu) \mu$, this can hold only for the very special case $\delta=\mu$ and $\nu=0$ (that is, if all necrotic material is inhibitive and is completely broken down). Numerical investigations indicate that there are two branches of solution, with the Major branch consisting of travelling wave and steady-state solutions and the Minor branch of steadystate solutions only. In the earlier models, steady-state solutions were restricted to the line $\delta-(1-\nu) \mu=\lambda$; now, with the addition of the cell shedding term, they exist over a range of parameter values. In summary we have

$\lambda>\mathbf{0}$ : Travelling waves and steady-states solutions exist over a range of parameters provided that $\delta-(1-\nu) \mu>\lambda$.

$\lambda=0$ : Travelling wave solutions exist provided that $\delta-(1-\nu) \mu>0$. Steady-state solutions can only exist if $\delta=\mu$ and $\nu=0$, in which case they are non-unique, being dependent on the initial conditions. 
From (15), we note that without the presence of inhibitor $(\mu=0)$, steady-state solutions exist in the range $\delta>\lambda$. Moreover, unlike the model of Ward and King (1999b). (15) is not a strict bound, the numerics indicating that the range in which only trivial long time solutions exist is larger than $\delta-(1-\nu) \mu \leq \lambda$.

\subsection{Numerical Results}

The derivation of the long time equations and boundary conditions and the numerical procedures are described in Ward and King (1999b). In each case the problem is formulated as a two-point boundary value problem and rescaled to a coordinate $y$ defined on the unit domain. The only alterations to the travelling wave system given in Ward and King (1999b) concern the boundary conditions

$$
\begin{aligned}
v(1)= & U+2 \Theta k_{m}\left(1,1-N_{1}-H_{1}, H_{1}\right) N_{1}, \\
\gamma(1)= & {\left[Q_{p}\left(1-p_{0}-N_{1}-H_{1}\right)-(v(1)-U)\right.} \\
& \left.\times\left(1-N_{1}-H_{1}\right)\right] / D_{p}-\Phi(1), \\
\Phi(1)= & {\left[Q_{h}\left(h_{0}-H_{1}\right)+(v(1)-U) H_{1}\right] / D_{h}, }
\end{aligned}
$$

where $\gamma=d n / d y$ and $\Phi=d h / d y$, and for each of the steady-state and bifurcation cases the modified boundary conditions are

$$
\begin{aligned}
v(1)= & 2 \Theta k_{m}\left(1,1-N_{1}-H_{1}, H_{1}\right) N_{1} F\left(S_{\infty}\right), \\
\gamma(1)= & {\left[Q_{p}\left(1-p_{0}-N_{1}-H_{1}\right)-v(1)\left(1-N_{1}\right.\right.} \\
& \left.\left.-H_{1}\right)\right] / D_{p}-\Phi(1), \\
\Phi(1)= & {\left[Q_{h}\left(h_{0}-H_{1}\right)+v(1) H_{1}\right] / D_{h}, }
\end{aligned}
$$

where the constants $N_{1}=n(1), H_{1}=h(1)$ and $U$ need to be determined as part of the solution. The boundary value problems are solved using the shooting and matching technique incorporated in the NAG routine D02AGF. The continuation procedure described in Ward and King (1999a) is used for the investigations in parameter space. Apart from the set of parameters used for Figure 11, all of the results to follow use parameter sets that lead to Regime I solutions when $\Theta=0$.

Figures 5-8 study the effects of the parameter $\Theta$ on various aspects of the long time growth behaviour, using the same data used for Figure 2. The existence of two possible long time outcomes is demonstrated in Figure 5, where the solid and dashed curves are the Major and Minor branches, respectively. In this

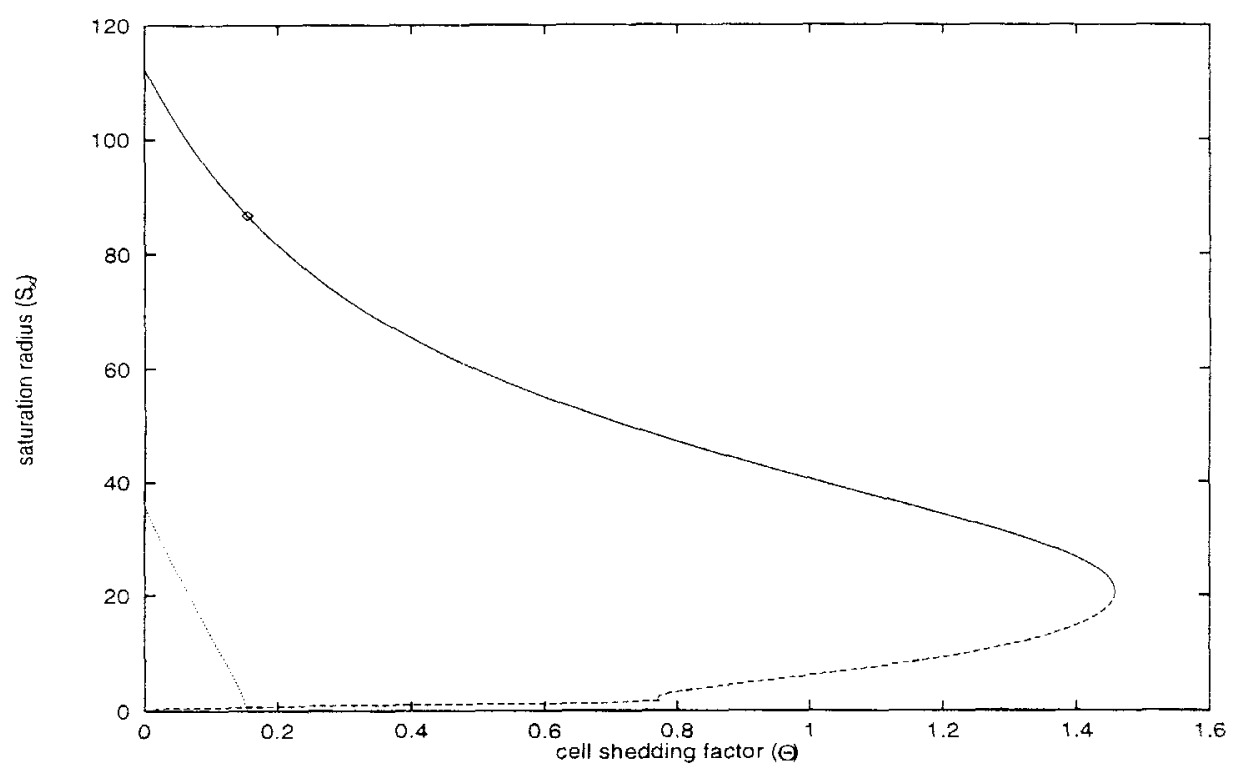

FIGURE 5 Effects of the cell shedding factor $\Theta$ on spheroid saturation size (solid $S_{\infty}^{M}$ and dashed $S_{\infty}^{m}$ ) and neerotic core radius (dots), defined as the position of the necrotic interface $\left(R_{\infty}\right)$. The position of the fully/partially necrotic core bifurcation is indicated by the $\odot$. The solid and dashed curves are the Major and Minor branches, respectively. 


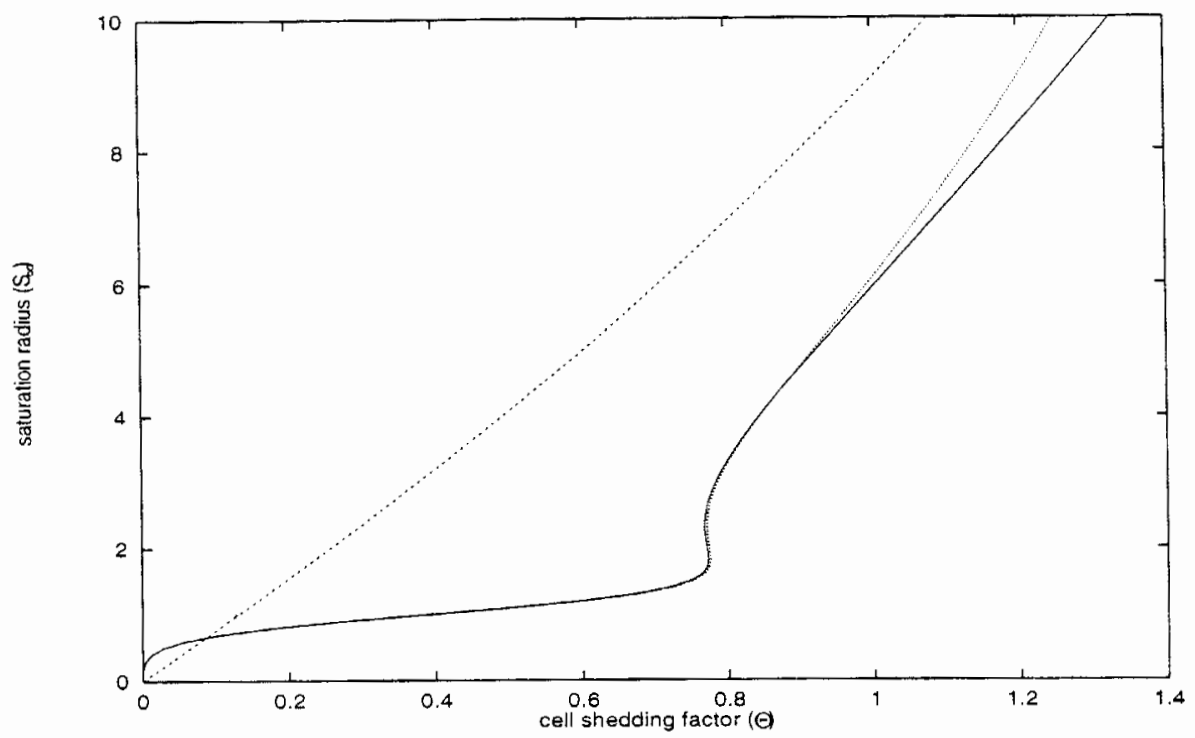

FIGURE 6 Plots of the Minor branch saturation size against $\Theta$, where the solid and dotted curve are asymptotic and numerical solutions, respectively, using $F(S)=1-2 / S+4 / 3 S^{2}$. The dashed curve is the asymptotic solution using $F(S)=1$. The asymptotic limit is $\beta_{1}, \beta_{2} \rightarrow 0$ and $D_{p}, D_{h} \rightarrow \infty$ and the numerical (dotted) curve is a blow-up of part of Figure 5 .

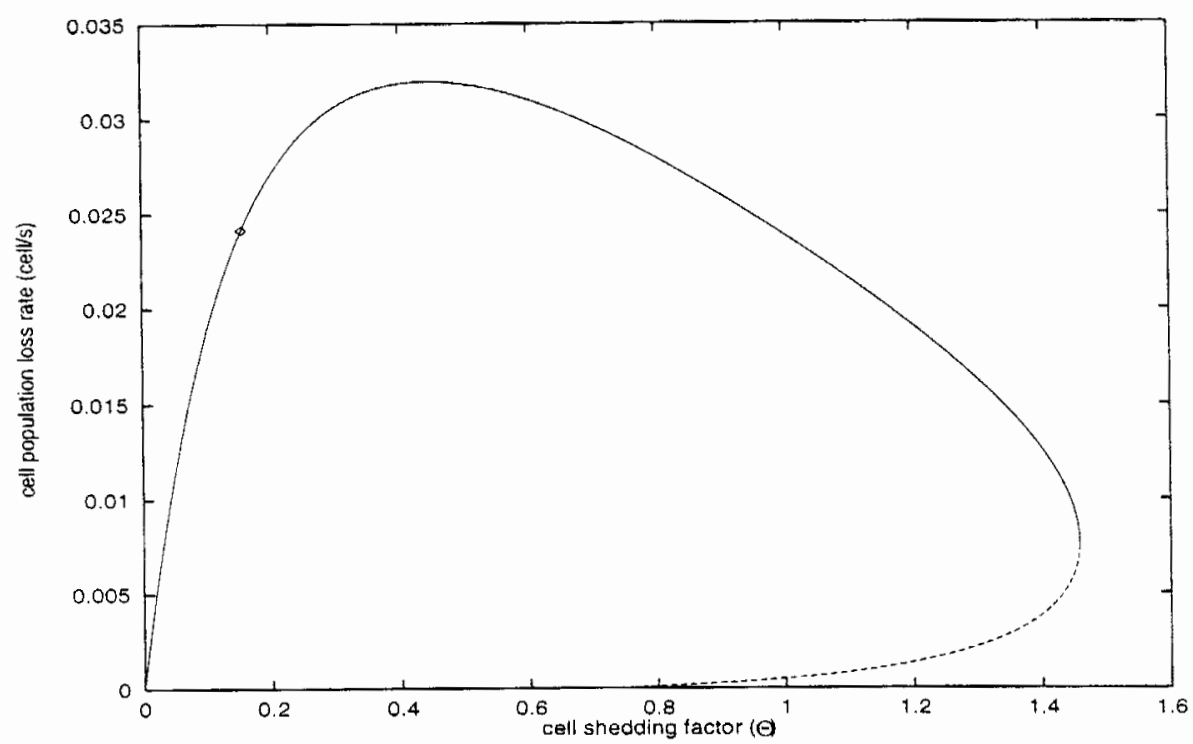

FIGURE 7 Cell population loss rate (cells/s) as a function of the cell shedding factor $\Theta$. The $\diamond$ denotes the fully/partially necrotic core bifurcation. The solid and dashed curves are the Major and Minor branches, respectively.

case, the Major/Minor branch bifurcation occurs at $\Theta \approx 1.46$; for values of $\Theta$ beyond this the cell shedding term is too strong for there to be any non-trivial solutions. The solid curve illustrates the (expected) effect that the saturation size decreases with increasing $\Theta$, the fully necrotic core solutions to the left of the ' $\diamond$ ' being larger than the partially necrotic solutions. In this case, the Minor branch consists only of partially necrotic solutions and shows the opposite behaviour of saturation size increasing with $\Theta$, before reaching the Major/Minor branch bifurcation. Since the Minor branch is unstable this behaviour suggests that as $\Theta$ increases there is an increase in the initial 'nucleation' size a spheroid must be in order for it to 


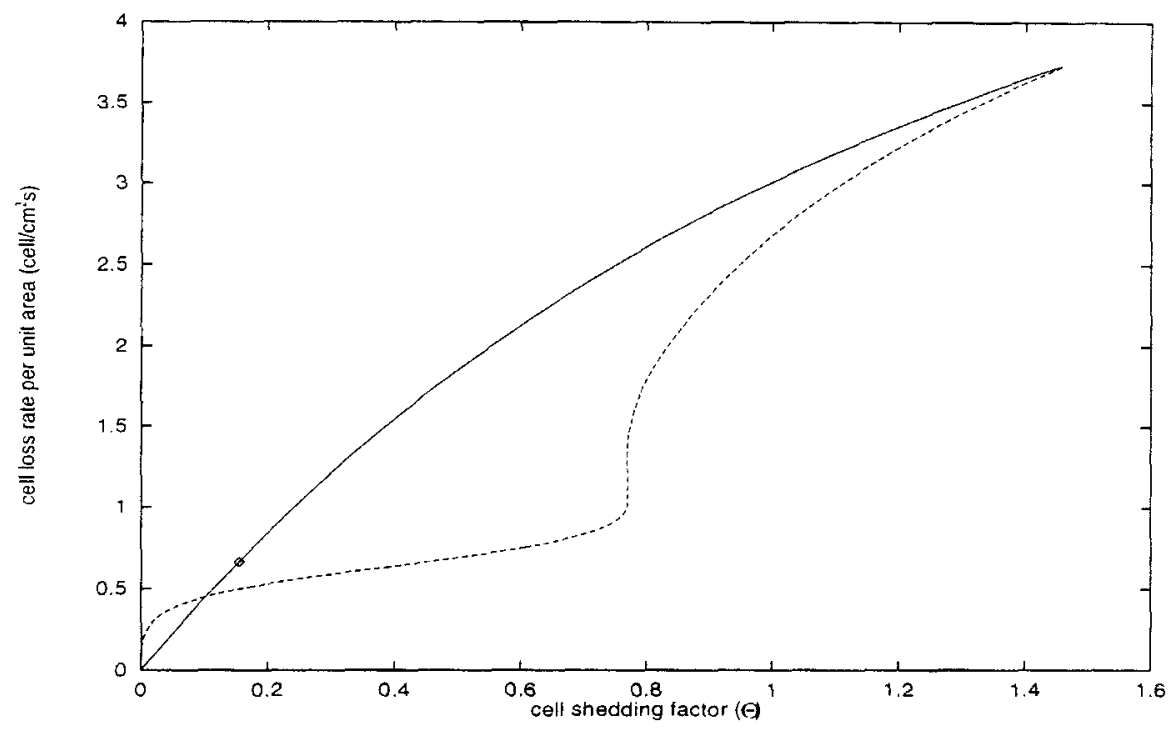

FIGURE 8 The effects of the cell shedding factor $\Theta$ on the cell loss rate per unit area (in cells $/ \mathrm{cm}^{2} \mathrm{~s}$ ). The solid and dashed curves are the Major and Minor branches, respectively.

attain a non-trivial long time solution. This is again to be expected, as is the existence of such a critical spherical size: the cell shedding rate depends on the surface area (and hence $S^{2}$ ) whereas in the early stages of growth the total mitotic rate depends on the volume (and hence $S^{3}$ ); the former dominates if $S$ is sufficiently small and the latter if it is sufficiently large. We note in Figure 5 that up to about $\Theta=0.75$, at least, the lower branch lies in the range of tumour radius for which a continuum model is not valid; in this regime the predicted 'nucleation' size will be only a few cells (at most) so in practice spheroids of any initial size would be expected to reach the Major branch radius $S_{\infty}^{M}$ in this regime.

Seeking solutions along the (non-physical) Minor branch as $\Theta \rightarrow 0, S_{\infty}^{m} \rightarrow 0$ (i.e. in Regime I), we find that $c \sim 1, h \sim h_{0}, n \sim 1-p_{0}-h_{0}$ and

$$
S_{\infty}^{m} \sim \Theta^{1 / 3}\left(\frac{8 k_{m}\left(1, p_{0}, h_{0}\right)}{a\left(1, p_{0}, h_{0}\right)}\right)^{\frac{1}{3}}
$$

using $F(S) \sim 4 / 3 S^{2}$ as $S \rightarrow 0$. We note that positivity of $\Theta$ implies that we need $a\left(1, p_{0}, h_{0}\right)>$ 0 , i.e. the birth rate must exceed the death rate. For $\Theta=0, a\left(1, p_{0}, h_{0}\right)=0$ marks the bifurcation between Regime I $\left(a\left(1, p_{0}, h_{0}\right)>0\right)$ and Regime II $\left(a\left(1, p_{0}, h_{0}\right)<0\right)$ solutions, as discussed in Section 2.4. An example of the behaviour using a parameter set giving Regime II solutions for $\Theta=0$ is shown later. Greater insight into the nature of the Minor branch can be gleaned by examining the case of small consumption rate $\left(\beta_{1}, \beta_{2} \rightarrow 0\right)$ and large diffusivities $\left(D_{p}, D_{h} \rightarrow \infty\right)$ for $S_{\infty}^{m}=O(1)$; this limit is relevant to Figure 5 in which $\beta_{1}=0.01, \beta_{2}=0$ and $D_{p}, D_{h}=300$. Omitting the details of the analysis, it can be shown that for $S_{\infty}^{m}=O(1)$ we have $c \sim 1$, $n \sim \tilde{n}\left(S_{\infty}^{m}\right), h \sim \tilde{h}\left(S_{\infty}^{m}\right), v \sim \tilde{a}\left(S_{\infty}^{m}\right) r / 3$ and

$$
\Theta \sim \frac{\tilde{a}\left(S_{\infty}^{m}\right)}{6 \tilde{k}_{m}\left(S_{\infty}^{m}\right)} \frac{S_{\infty}^{m}}{F\left(S_{\infty}^{m}\right)},
$$

where $\tilde{a}\left(S_{\infty}^{m}\right)=a\left(1,1-\tilde{n}\left(S_{\infty}^{m}\right)-\tilde{h}\left(S_{\infty}^{m}\right), \tilde{h}\left(S_{\infty}^{m}\right)\right)$ and $\tilde{k}_{m}\left(S_{\infty}^{m}\right)=k_{m}\left(1,1-\tilde{n}\left(S_{\infty}^{m}\right)-\tilde{h}\left(S_{\infty}^{m}\right), \tilde{h}\left(S_{\infty}^{m}\right)\right)$. The asymptotic solutions for this limit are compared with the numerical solution in Figure 6, demonstrating that the analysis predicts the slight fold seen in the numerical curve. Also shown in Figure 6 is the asymptotic solution for $F(S)=1$, showing that similar qualitative results occur for larger $S_{\infty}^{m}$; the folds in the other curves result from the behaviour of $F(S)=$ $1-2 / S+4 / 3 S^{2}$ for $S=O(1)$ and are not of physical significance.

Using Equation (4) and converting to dimensional quantities, as before, the cell population loss rate (in cell/s) as a function of $\Theta$ is shown in Figure 7 , the 
Major and Minor branches forming a closed loop. To the left of the maximum on the Major branch, the increasing proportion of detached cells results in the increase in the shedding rate. However, as $\Theta$ becomes larger, causing the reduction in size of the spheroid and consequently of the number of cells at the surface, there is a decrease in the cell loss rate towards the Major/Minor branch bifurcation. In Figure 8 the cell loss rate per unit area is monotonically increasing in $\Theta$ for both Major and Minor solutions, as is to be expected. Again, we have converted to dimensional quantities, so that a comparison can be made with experimentally observed values, ranging from $0.1-6$ cells $/ \mathrm{cm}^{2}$ s (Sasaki et al., 1984; Kohno et al., 1986; Landry et al., 1981). In the limit of $\Theta \rightarrow 0$ with $S_{\infty}^{m} \rightarrow 0$ (see (17)), the cell flux at the surface can be shown to be $O\left(\Theta^{1 / 3}\right)$, explaining the rapid initial increase on the Minor branch. The abrupt increase further along this curve corresponds to the fold in Figure 6.

The paths of the non-trivial/trivial (solid curve), travelling wave/steady-state (dashed curve) and fully/partially necrotic core (smaller-dashed curve) bifurcations in $\Theta$ and $D_{p}=Q_{p}$ parameter space are shown in Figure 9 for the Cell shedding-no inhibitor model. Here, the parameters are given by (7), except that $\mu=0$ so that there is no inhibitor present, and we set $\lambda=0.8$ (rather than $\lambda=1$ ) so that the cross-over of the bifurcation curves is emphasised. The solid curve was generated by tracking along $\partial \Theta / \partial S_{\infty}=0$, which is where the Major and Minor branches meet, as $D_{p}=Q_{p}$ is varied. The solid curve is started at about $D_{p}=70$, due to the failure of the numerical procedure for smaller diffusivities (the evaluation of the curve $\partial \Theta / \partial S_{\infty}=0$, if it exists, being very sensitive to the initial guess there). However, it is believed that for smaller diffusivities the solid curve tracks along the dashed curve. Below the dashed curve, the Major branch consists of travelling wave solutions. To the right of the cross-over point of the dashed and smaller-dashed bifurcation curves, fully necrotic core solutions exist between those curves and are Major branch solutions. The fully necrotic core solutions to the left of the cross-over point lie above the smaller-dashed curve and are Minor branch solutions, except for those that exist above the dashed curve in the vicinity of the cross-over point.

Figure 10 shows the effect of $\Theta$ on the saturation size along the dotted paths labelled $\mathbf{A}$ and $\mathbf{B}$ in Figure 9. The Major branch along path $\mathbf{B}$ descends rapidly from the travelling wave/steadystate bifurcation on increasing $\Theta$, until it reaches the

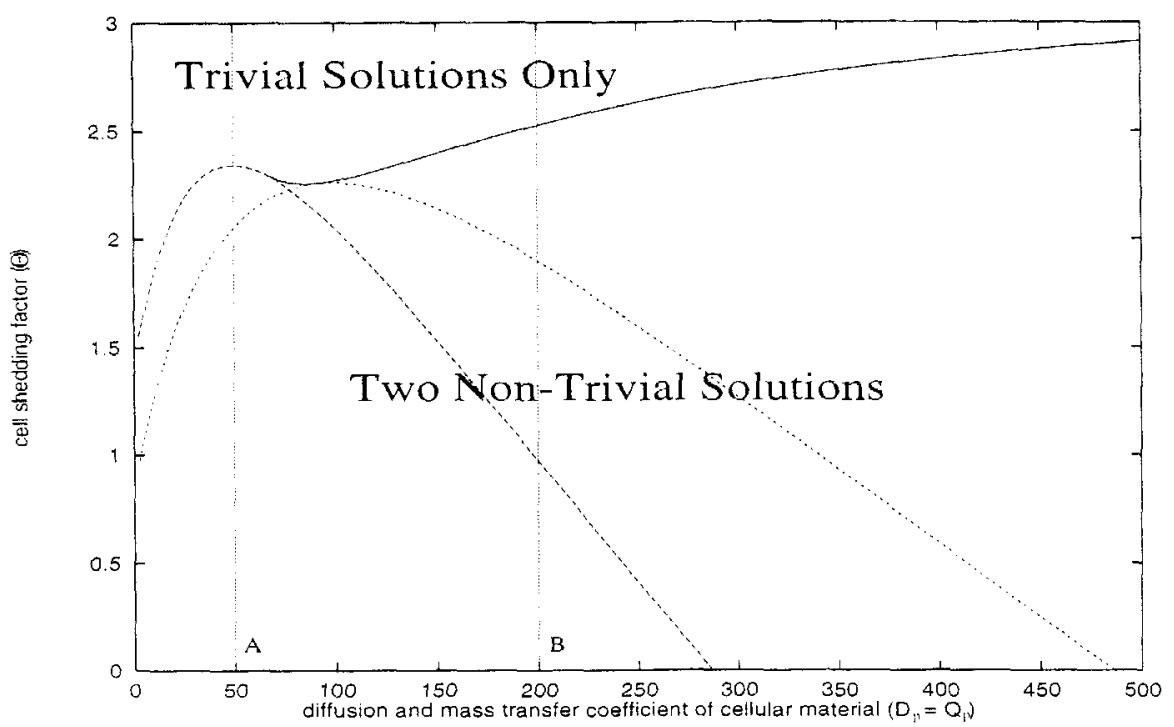

FIGURE 9 The paths of the travelling wave/steady-state (dashed) and fully/partially necrotic core (shorter-dashed) bifurcations in $D_{p}=Q_{p}$ and $\Theta$ parameter space. The solid curve tracks the path of $\partial \Theta / \partial S_{\infty}=0$, i.e. the bifurcation between the Major and Minor branches, above which only trivial solutions exist. The paths for Figure 10 are indicated by the dotted lines labelled $\mathbf{A}$ and $\mathbf{B}$. 


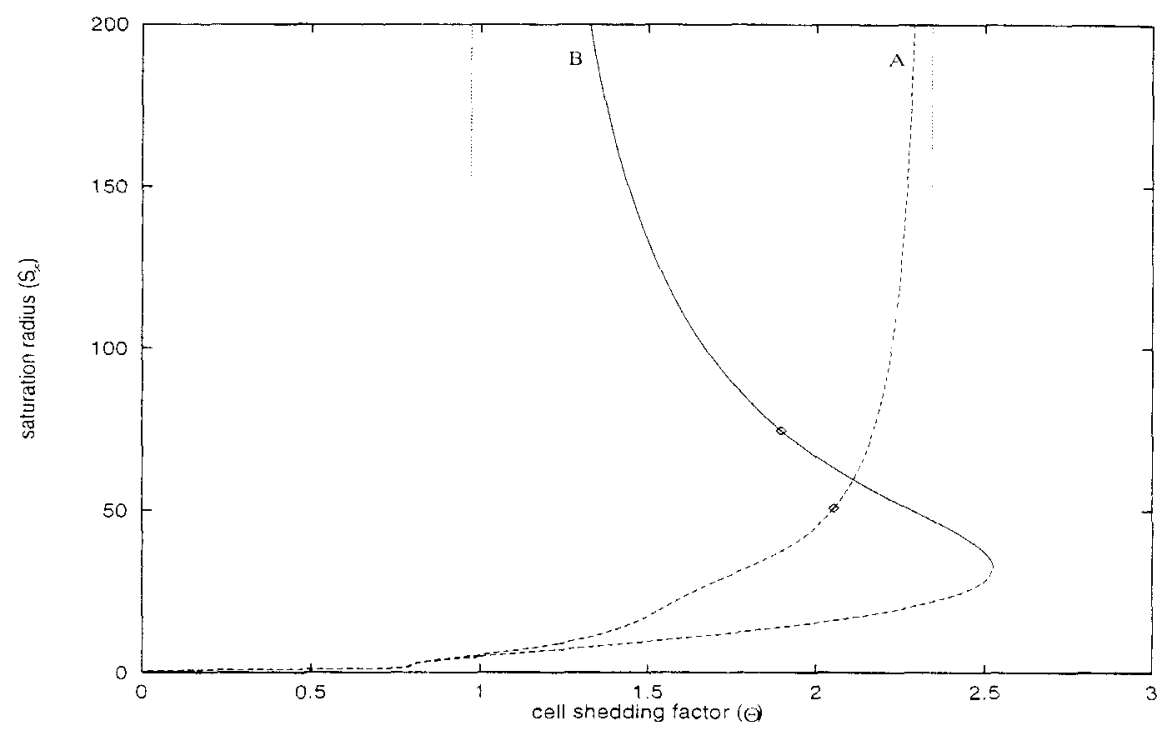

FIGURE 10 Saturation size (solid and dashed curve) against $\Theta$ along paths $\mathbf{A}$ and $\mathbf{B}$ of Figure 9. Major branch solutions are depicted by the solid curve and Minor branch solutions by the dashed curves. Travelling wave solutions (not shown) exist to the left of dashed lines at the top of the figure, marking the location of the travelling wave/steady-state bifurcation. The fully necrotic core solutions occuring for $\Theta$ between the dotted line and the $\diamond$ marking the fully/partially necrotic corc bifurcation.

Major/Minor branch bifurcation at $\Theta_{c} \approx 2.53$. As in Figure 5, the Minor branch consists only of partially necrotic steady-state solutions, with saturation size increasing in $\Theta$ to $S_{\infty}^{m} \approx 33$. Along path $\mathbf{A}$ all steadystate (including fully necrotic core solutions) exist on the Minor branch, on which the saturation size is monotonically increasing in $\Theta$, blowing up as $\Theta$ approaches travelling wave/steady-state bifurcation point. We note that the travelling wave solutions exist to the left of travelling wave/steady-state bifurcation point indicated by the dashed lines in Figure 10 and are Major branch solutions.

The saturation radius is plotted against $\Theta$ in Figure 11 for a parameter set giving Regime II solutions if $\Theta$ is set to zero. The parameters are given by (7) with $\lambda=0.4, D_{p}=Q_{p}=250, \mu=0$ (so that there is no inhibitor present) and $p_{0}=0$, implying $a\left(1, p_{0}, h_{0}\right)<0$. Here, the two saturation sizes satisfying $\Theta=0$ correspond to those occurring for $\lambda=0.4$ on curve $\mathbf{B}$ in Figure 18 of Ward and King (1990a). The figure shows that the Minor Branch solution curve tends to the lower of the two Regime II solutions in the limit $\Theta \rightarrow 0$ (rather than towards the trivial solution, i.e. unlike Figures 5, 10). This is consistent with the analysis leading to Equation (17), demonstrating the non-existence of non-trivial solutions with $S_{\infty}^{m} \rightarrow 0$ in the limit $\Theta \rightarrow 0$ for parameter sets that give rise to Regime II solutions when $\Theta=0$. The Minor branch solutions are again expected to be unstable, with the Major branch and trivial solutions being the long-time attractors.

In Figure 12 we examine the effects of $\Theta$ on the travelling wave/steady-state bifurcation curves in $D_{p}=Q_{p}$ and $\lambda$ parameter space. This continues a study in Ward and King (1999b), the parameter set being given by (7), except for $D_{h}=Q_{h}=400, P=$ 0.9 and $\mu=0.25$. The increase in the cell shedding flux that results from increasing $\Theta$ can be seen to lead to a decrease in the number of cells in the spheroid, thus shrinking the travelling wave regions. The nonmonotonicity of the curves is due to the shifting of the dominant cellular material loss mechanism from consumption to leakage on increasing $D_{p}=Q_{p}$.

\section{DISCUSSION}

The process of cell shedding has largely been ignored in multicell spheroid growth models, despite the weight of evidence that suggests it is an important factor in avascular growth, at least in vitro 


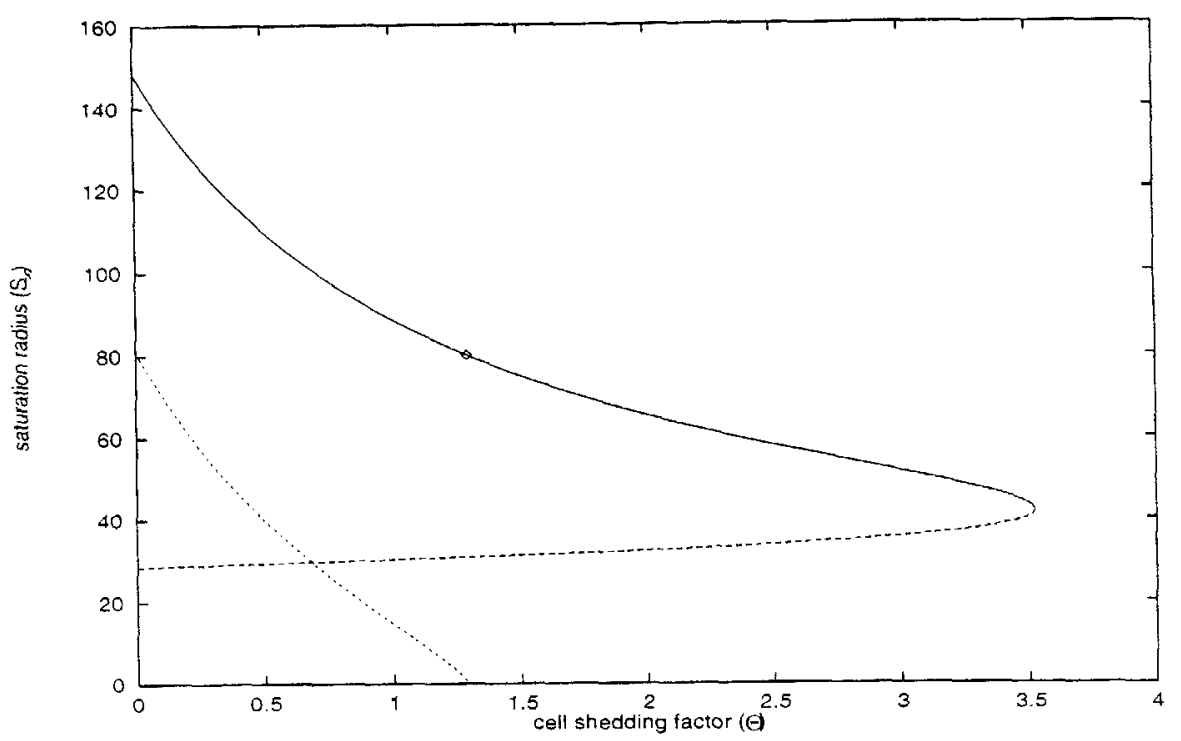

FIGURE 11 Saturation size (solid and dashed curve) and necrotic core radius $R_{\infty}$ (smaller dashed curve) against $\Theta$, for a parameter set of Regime II when $\Theta=0$. The fully necrotic core solutions lie on the solid line for $\Theta$ between 0 and the $\diamond$.

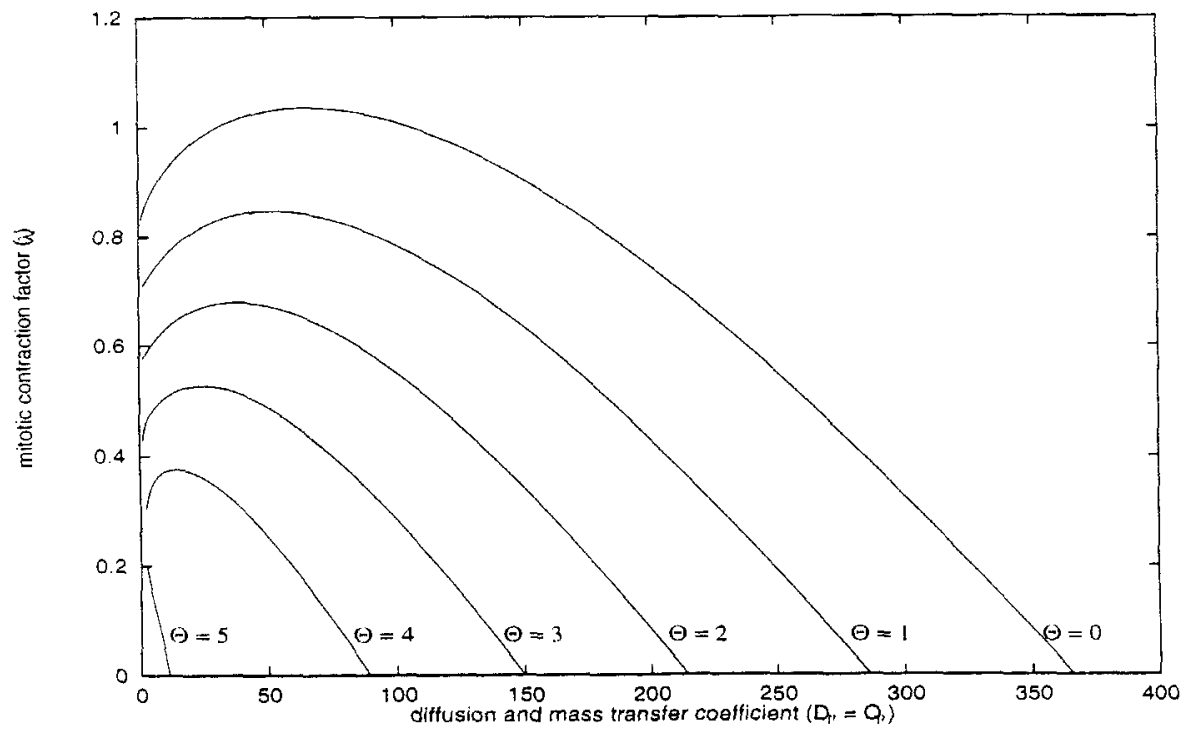

FIGURE 12 The effects of the cell shedding factor $\Theta$ on the travelling wave/steady-state bifurcation in $D_{p}=Q_{p}$ and $\lambda$ space.

(Landry et al., 1981; Kohno et al., 1986; Sasaki et al., 1984; Weiss, 1978). In this paper the model of Ward and King (1999b) has been adapted to include a cell shedding term so that its effect can be investigated. Numerical results shown in Section 3 and 4 demonstrate that the cell shedding process has significant quantitative effects, even for fairly weak shedding rates (see, for example, Figures 2 and 5).
Furthermore, as shown in Figure 3, the qualitative behaviour may be affected too, the addition of cell shedding leading to growth saturation (as opposed to continual growth) for sufficiently high cell shedding rates (i.e. sufficiently large $\Theta$ ). The model continues to mimic successfully experimentally observed growth behaviour and tumour heterogeneity when cell shedding is included. 
The choice of cell shedding rate function $K(c, n, p, h)=\Theta k_{m}(c)$ means that the mechanism can be modelled with the addition of only one extra parameter $\Theta$, values for which can be obtained from published experimental data (indeed, it is one of the quantities for which good experimental information is available, making an assessment of its effects on the behaviour predicted by the model particularly worthwhile). The addition of the cell shedding term gives rise to two non-trivial branches of long time solutions, which vary smoothly with $\Theta$ to form two curves we have termed Major and Minor branches. In our earlier models, the existence of two nontrivial long time solutions was only observed in rather restricted parameter regimes. Beyond a critical value $\Theta_{c}$, where the Major and Minor branches meet, there are no non-trivial solutions due to the cell shedding rate being too strong, forcing spheroid extinction; cell lines with $\Theta>\Theta_{c}$ will therefore be unable to form spheroids. The effect of strengthening the cell shedding rate on the (stable) Major Branch solutions is to decrease the travelling wave speed or saturation size of the spheroid and to expand the parameter regimes in which saturation occurs. Along the Minor branch, consisting only of steady-states solutions, the saturation size increases with $\Theta$; these solutions are expected to be unstable and this behaviour reflects the need on increasing $\Theta$ for a larger initial spheroid size in order for the spheroid to be viable. The expected instability of the Minor branch solutions is confirmed by the stability analysis in Appendix B and is illustrated in Figure 4 . We thus expect the long time solutions on the Major branch to be the physical ones. We note that in regions in which only trivial long time solutions exist the cell shedding rate is too strong for the cells to grow as spheroid cultures. Analysis of the long time equations demonstrates that, except in a physically implausible case of zero dead cell volume $(\delta=0)$, the process of cell shedding and mitotic inhibition together cannot cause growth saturation; i.e. some non-negligible level of leakage or consumption of cellular material is required for saturation to be achieved. In summary, the analysis demonstrates that necessary conditions for growth saturation to occur are either: i) Leakage of cellular material and/or inhibitor (i.e. $Q_{p}, Q_{h}>0$ ),

or ii) Cellular material consumption and cell shedding (i.e. $\lambda, \Theta>0$ ).

We note from $i i$ ) that, in contrast to our previous models, steady-state solutions do exist over a range of parameters in the case when the cellular material is prevented from leaking out. We note further that, since $K(c, n, p, h)$ must be positive to be biologically appropriate, conditions $i$ ) and $i$ ) both hold for any suitable choice of $K(c, n, p, h)$.

As already indicated, for experiments in which the cell shedding rate can be determined, the value of $\Theta$ can readily be calculated using Equation (6), the values of $n(S, t), \tilde{k}_{m}$ and $r_{0}$ being comparatively easy to determine experimentally. An interesting experiment would be to study spheroid growth in a medium containing some chemical that promotes cell shedding, so features such as growth rate and saturation size can be determined as a function of the cell shedding rate; an example of such a chemical is trypsin, which is often used to dissociate cells from spheroids (for example Freyer and Sutherland, 1980). Comparison of such experiments with the model predictions would provide a valuable (quantitative) means to assess the modelling assumptions.

All the comments above regarding stability concern stability to radially symmetric perturbations. There is currently (e.g. Greenspan, 1976; Chaplain and Sleeman, 1993; Byrne and Chaplain, 1996) widespread interest in issues of stability to non-radial perturbations (with reference to the formation of 'hot spots' for example; see Chaplain, 1995). We therefore also note that cell shedding is likely to be a stabilising influence on such non-radially symmetric perturbations, since if a part of the surface grows more rapidly than surrounding regions the increase in surface area there will lead to a local enhancement of cell shedding.

We have shown that the inclusion of even fairly low levels of cell shedding leads to quite significant changes in the quantitative behaviour, from which we conclude that in order to simulate the growth of multicell spheroids accurately the effects of cell shedding must be considered. Although it has been 
shown that cell shedding is an important cell loss mechanism in vivo, the situation in vivo is unclear. It is anticipated that cells detached from a tumour body will to a large extent be prevented from moving far by neighbouring normal cells, and will thus remain in the locality of the primary tumour and could conceivably rejoin it. The number of cells that escape permanently via the vasculature or via lymph vessels, thus being able to form secondary growths (metastases), is likely to be relatively low. The mathematical modelling of the behaviour in vivo of tumour cells after they have been shed thus presents a significant, and worthwhile, challenge.

\section{Appendix A: Model equations}

A full description of model derivation and nondimensionalisation is given in Ward and King (1999b); we give only a brief summary here. The main variables in the model are live cell density $n$, cellular material concentration $p$, inhibitor concentration $h$, nutrient concentration $c$, velocity $v$ and the moving boundary marking the tumour boundary $S$, the independent variables being time $t$ and the radial coordinate $r$. The model is presented below in non-dimensional form; it is worth noting that time is scaled with the maximum rate of cell birth and space is scaled with the size of a single cell. The dimensionless form for the no void condition (1) is

$$
n+p+h=1
$$

whereby using $p=1-n-h$ the equation for $p$ can be decoupled. The modelling is based on a continuum of live cells which divide or die at rate depending on the concentrations of nutrient, cellular and inhibitive material. We assume that the cell-cell contacts are sufficiently strong that the effects of random motion and chemotaxis are negligible. The nutrient is assumed to diffuse from the surface and to be consumed by the living cells, the timescales for these processes being significantly shorter than the cell birth rate. The inhibitive material is diffusive and generated from the products of cell death; it can be broken down by the living cells into cellular material.
The changes in volume by cell birth and death and by the diffusion of cellular and inhibitive material causes movement within the tumour which is described by a velocity field, leading to the convective terms in the equations below. Using these assumptions and adopting spherical symmetry, the full system of dimensionless equations is

$$
\begin{aligned}
& \frac{\partial n}{\partial t}+\frac{1}{r^{2}} \frac{\partial\left(r^{2} v n\right)}{\partial r}=a(c, 1-n-h, h) n \\
& \frac{1}{r^{2}} \frac{\partial}{\partial r}\left(r^{2} \frac{\partial c}{\partial r}\right)=k(c, 1-n-h, h) n \\
& \frac{\partial h}{\partial t}+\frac{1}{r^{2}} \frac{\partial\left(r^{2} v h\right)}{\partial r}=\frac{D_{h}}{r^{2}} \frac{\partial}{\partial r}\left(r^{2} \frac{\partial h}{\partial r}\right)+l(c, h) n-\frac{D_{p}}{r^{2}} \frac{\partial}{\partial r}\left(r^{2} \frac{\partial n}{\partial r}\right) \\
& \frac{1}{r^{2}} \frac{\partial\left(r^{2} v\right)}{\partial r}= b(c, 1-n-h, h) n \\
&+\frac{D_{h}-D_{p}}{r^{2}} \frac{\partial}{\partial r}\left(r^{2} \frac{\partial h}{\partial r}\right)
\end{aligned}
$$

where the dimensionless diffusion coefficients of cellular material $D_{p}$ and inhibitor $D_{h}$ are assumed constant. We note the Equation (23) can be derived by summing Equations (20), (22) and the equivalent equation for $p$ and using (19). The dimensionless functions $a, b, k$ and $l$ can be interpreted as being the net rates of birth, volume change, nutrient consumption and inhibitor production and are given by

$$
\begin{aligned}
a(c, p, h)= & k_{m}(c, p, h)-k_{d}(c), \\
b(c, p, h)= & (1-\lambda) k_{m}(c, p, h)-(1-\delta) k_{d}(c) \\
& -(1-\nu) \psi h, \\
k(c, p, h)= & \beta_{1}\left(\frac{c^{m_{1}}}{c_{c}^{m_{1}}+c^{m_{1}}}\right)+\beta_{2} k_{m}(c, p, h), \\
l(c, h)= & \mu k_{d}(c)-\psi h,
\end{aligned}
$$

where $c_{c}, \lambda, \delta, \mu, \psi, \nu, \beta_{1}, \beta_{2}$ and $m_{1}$ are all constant. The mitotic rate $k_{m}$ and death rate $k_{d}$ functions are described below. In brief, $\lambda$ is dimensionless volume of cellular material consumed during mitosis, $\delta$ is the ratio of dead cell and live cell volumes, 
$\mu$ is the proportion of inhibitive material in a dead cell $(\mu \leq \delta), \psi$ is the rate of inhibitor breakdown and $\nu$ is the gain in volume of cellular material during breakdown of the inhibitor $(\nu \leq 1)$. The consumption rate expression $k$ includes both nutrient consumption by day-to-day cellular activity $\left(\beta_{1}>0\right)$ and an additional amount consumed during mitosis $\left(\beta_{2}>0\right)$. The birth rate and death rate expressions considered are

$$
\begin{aligned}
k_{m}(c, p, h)= & \left(\frac{c^{m_{1}}}{c_{c}^{m_{1}}+c^{m_{1}}}\right)\left(\frac{p^{m_{3}}}{p_{c}^{m_{3}}+p^{m_{3}}}\right) \\
& \times\left(1-P \frac{h^{m_{4}}}{h_{c}^{m_{1}}+h^{m_{4}}}\right), \\
k_{d}(c)= & \frac{B}{A}\left(1-\sigma \frac{c^{m_{2}}}{c_{d}^{m_{2}}+c^{m_{2}}}\right),
\end{aligned}
$$

where $B / A, c_{d}, p_{c}, h_{c}, \sigma, P$ are all constants with $\sigma \leq 1$ and $P \leq 1$. We note that $B / A$ is the ratio of the maximum death rate $B$ to the maximum birth rate $A$. The numerical solutions throughout this paper assumed that all the exponents $m_{i}=1$; however, the general forms of these functions have been included here so the model proposed is a natural extension to the model described in Ward and King (1997).

The dimensionless initial and boundary conditions are

$$
\begin{aligned}
& \text { at } t=0 \quad n=1, \quad h=0, \quad S=1, \\
& \text { at } r=0 \quad \frac{\partial c}{\partial r}=\frac{\partial n}{\partial r}=\frac{\partial h}{\partial r}=v=0, \\
& \text { at } r=S(t) \quad c=1, \\
& \quad \frac{d S}{d t}=v-2 \Theta k_{m}(1,1-n-h, h) F(S), \\
& D_{p}\left(\frac{\partial n}{\partial r}+\frac{\partial h}{\partial r}\right)=Q_{p}\left(1-n-h-p_{0}\right) \\
& \quad-\left(v-\frac{d S}{d t}\right)(1-n-h), \\
& D_{h} \frac{\partial h}{\partial r}=Q_{h}\left(h_{0}-h\right)+\left(v-\frac{d S}{d t}\right) h,
\end{aligned}
$$

where the mass transfer coefficients $Q_{p}$ and $Q_{h}$ and the external concentrations of cellular material $p_{0}$ and inhibitor $h_{0}$ are all assumed constant.
We note that the model contains a large number of dimensionless parameters, with little or no experimental data being available for a number of these; see Ward and King (1997, 1999a and 1999b) for the determination of estimates of parameter values.

\section{Appendix B: Stability of the Minor branch solutions for small spheroids}

In this Appendix we seek solutions of the transient model in the (non-physical) limit $\Theta \rightarrow 0$ in the vicinity of steady-state solutions along the Minor branch. The appropriate scaling for $S$ is given by (17) and we write $S=\Theta^{1 / 3} S^{*} \ll 1$. Following an initial transient, on the timescale $t=O\left(\Theta^{2 / 3}\right)$, in which there is negligible growth, the transient model equations give at leading order $c \sim 1, h \sim h_{0}$, $n \sim 1-p_{0}-h_{0}, v \sim a_{0} r / 3$, where $a_{0}=a\left(1, p_{0}, h_{0}\right)$. Noting that $F(S) \sim 4 / 3 S^{2}$, the leading order equation for spheroid growth is then

$$
\frac{d S^{*}}{d t} \sim \frac{a_{0}}{3} S^{*}-\frac{8 k_{m_{0}}}{3 S^{* 2}}
$$

where $\quad k_{m_{0}}=k_{m}\left(1, p_{0}, h_{0}\right)$. Defining $S_{\infty}^{*}$ $=\left(8 k_{m_{0}} / a_{0}\right)^{1 / 3} \sim \Theta^{-1 / 3} S_{\infty}$ for $a_{0}>0$, so that $S_{\infty}^{*}$ gives the leading order saturation size, it follows that to leading order

$$
S^{* 3}=S_{\infty}^{* 3}+\left(S_{0}^{* 3}-S_{\infty}^{* 3}\right) e^{a_{0} t},
$$

where $S_{0}^{*}=S^{*}(0)$. We note if we use $F(S)=1$ instead, then the appropriate rescaling is $S=\Theta S^{*}$ and the appropriate form for $S_{\infty}^{*}$ is $S_{\infty}^{*}=6 k_{m_{0}} / a_{0}$, resulting in $S^{*}=S_{\infty}^{*}+\left(S_{0}^{*}-S_{\infty}^{*}\right) e^{a_{0} t / 3}$; the discussion below also applies to this case.

For $a_{0}>0$ (Regime I) the steady-state $S_{\infty}=S_{\infty}^{*}$ is unstable; if $S_{0}^{*}>S_{\infty}^{*}$, so the initial size is greater than the steady-state, the spheroid will grow larger at an exponential rate (until $S$ becomes of $O(1)$ ). By contrast, if $S_{0}^{*}<S_{\infty}^{*}$ the spheroid becomes extinct at time $t \sim t_{E}$, given by

$$
t_{E}=\frac{1}{a_{0}} \ln \left(\frac{S_{\infty}^{* 3}}{S_{\infty}^{* 3}-S_{0}^{* 3}}\right),
$$

for $F(S)=1-2 / S-4 / 3 S^{2}$. In the case of $F(S)=$ 1 we instead have $t_{E}=3 \ln \left(S_{\infty}^{*} /\left(S_{\infty}^{*}-S_{0}^{*}\right)\right) / a_{0}$. For 
$a_{0}<0$ (Regime II) the Minor branch solution tends to steady-state of finite size as $\Theta \rightarrow 0$, and we have $S_{\infty}^{*}<0$ in (27); the above analysis shows that solutions with $S_{0}^{*}=O(1)$ die out in finite time, again indicating the Minor branch to be unstable.

\section{Acknowledgements}

The first author gratefully acknowledges the support of an EPSRC Earmarked Research Studentship and the second a Nuffield Foundation Science Research Fellowship.

\section{References}

Byrne, H. M. and Chaplain, M. A. J. (1996). Modelling the role of cell-cell adhesion in the growth and development of carcinomas. Math. Biosci., 135, 187-216.

Carlsson, J. (1977). A proliferation gradient in three-dimensional colonies of cultured human glioma cells. Int. J. Canc., 20, $129-136$.

Casciari, J. J., Sotirchos, S. V. and Sutherland, R. M. (1992). Mathematical modelling of microenvironment and growth in EMT6/RO multicellular tumour spheroids. Cell Prolif., 25, $1-22$.

Chaplain, M. A. J. (1995). Reaction-diffusion prepatterning and its potential role in tumour invasion. J. Biol. Syst., 3, 929-936.

Chaplain, M. A. J. and Sleeman, B. D. (1993). Modelling the growth of solid tumours and incorporating a method for their classification using non-linear elasticity theory. J. Math. Biol., 31, 431-473.

Congar, A. D. and Ziskin, M. C. (1983). Growth of mammalian multicellular tumour spheroids. Canc. Res., 43, 558-560.

Folkman, J. and Hochberg, M. (1973). Self-regulation of growth in three dimensions. J. Exp. Med., 138, 745-753.

Freyer, J. P. Schor, P. L. and Saponara, A. G. (1988). Partial purification of a protein-growth inhibitor from multicell spheroids. Biochem. Biophys. Res. Comm., 152, 463-468.

Freyer, J. P. and Sutherland, R. M. (1980). Selective dissociation and characterization of cells from different regions of multicell tumour spheroids. Canc. Res., 40, 3956-3965.

Freyer, J. P. and Sutherland, R. M. (1986). Regulation of growth saturation and development of necrosis in EMT6/RO multi- cellular spheroids glucose and oxygen supply. Canc. Res., 46, 3504-3512.

Greenspan, H. P. (1976). On the growth and stability of cell cultures and solid tumours. J. Theo. Biol., 56, 229-242.

Haji-Karim, M. and Carlsson, J. (1978). Proliferation and viability in cellular spheroids of human origin. Canc. Res., 38, $1457-1464$

Harel, L., Chatelain, G. and Golde, A. (1984). Density dependent inhibition of growth: inhibitory diffusible factors from 3T3 and Rous sarcoma virus (RSV)-transformed 3T3 cells. $J$. Cell Phys., 119, 101-106.

Inch, W. R., McCredie, J. A. and Sutherland, R. M. (1970). Growth of nodular carcinomas in rodents compared with multicell spheroids in tissue culture. Growth, 34, 271-282.

Iversen, O. H. (1991). The hunt for endogenous growth inhibitory and or tumour supression factors - their role in physiological and pathological growth regulation. Adv. Canc. Res., 57, 413-453.

Kohno, N., Ohnuma, T., Biller, H. and Holland, J. F. (1986). Cell shedding from the surface of multicellular tumor spheroids (MTS) and inhibition by anticancer agents. Proc. Am. Ass. Canc. Res., 27, 388.

Landry, J., Freyer, J. P. and Sutherland, R. M. (1981). Shedding of mitotic cells from the surface of multicell spheroids during growth. J. Cell Physiol., 106, 23-32.

Landry, J.. Freyer, J. P. and Sutherland. R. M. (1982). A model for the growth of multicellular spheroids. Cell Tiss. Kinet., 15 , $585-594$.

Sasaki, T., Yamamoto, M., Yamaguchi, T. and Sugiyama, S. (1984). Development of multicellular spheroids of HeLa cells cocultured with fibroblasts and their response to X-Irradiation. Canc. Res., 44, 345-351

Shymko. R. M. and Glass, L. (1976). Cellular and geometric control of tissue growth and mitotic instability. J. Theo. Biol. 63, 355-374.

Ward, J. P. (1997). Mathematical modelling of avascular tumour growth. PhD Thesis, Nottingham University.

Ward, J. P. and King, J. R. (1997). Mathematical modelling of avascular-tumour growth. IMA J. Math. Appl. Med, 14, $39-69$.

Ward, J. P. and King, J. R. (1999a). Mathematical modelling of avascular-tumour growth II: modelling growth saturation. IMA J. Math. Appl. Med, 16, 171-211.

Ward, J. P. and King, J. R. (1999b). Mathematical modelling of the effects of mitotic inhibitors on avascular tumour growth. J. Theor. Med., 1, 287-311.

Weiss, L. (1978). Some mechanisms involved in cancer cell detachment by necrotic material. Int. J. Canc, , 22, 196-203. 


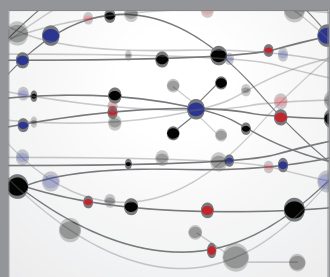

The Scientific World Journal
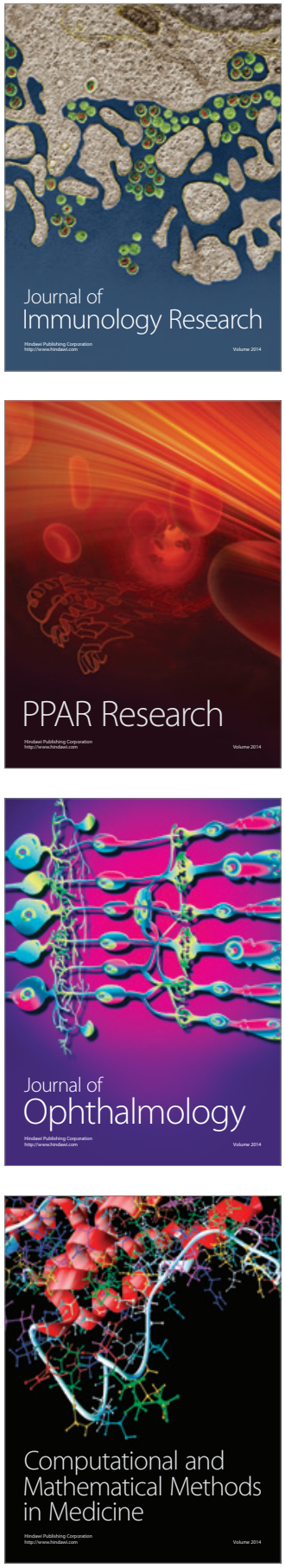

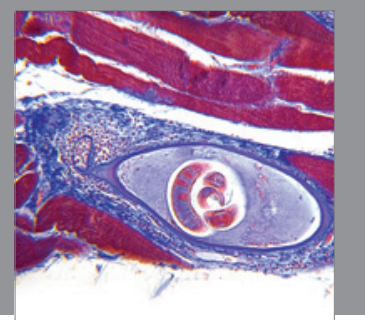

Gastroenterology

Research and Practice
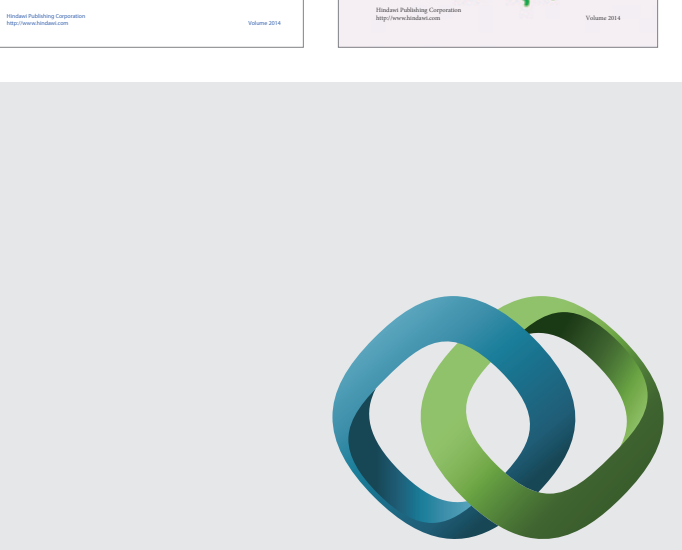

\section{Hindawi}

Submit your manuscripts at

http://www.hindawi.com
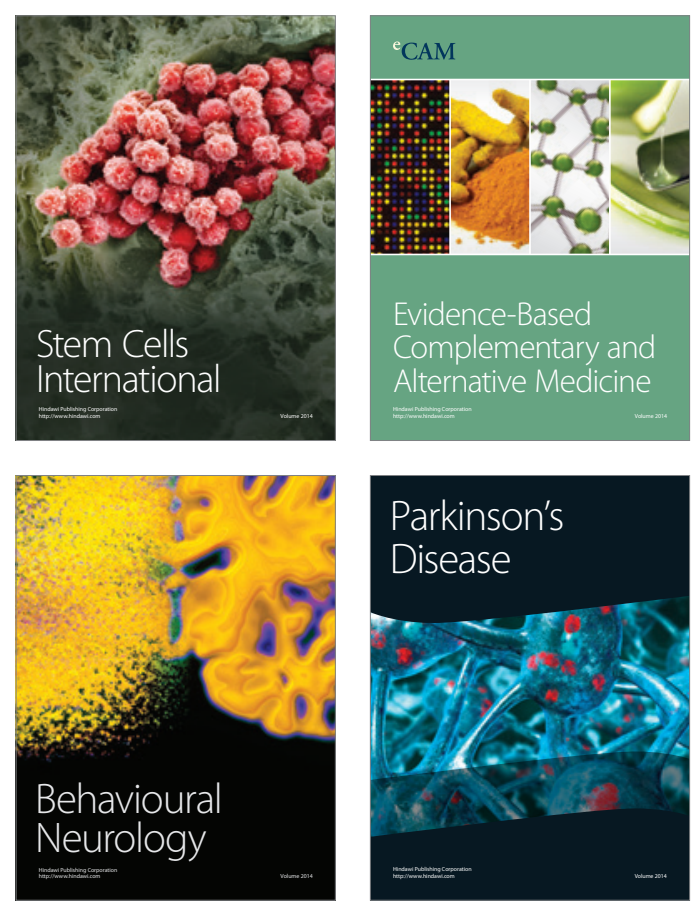

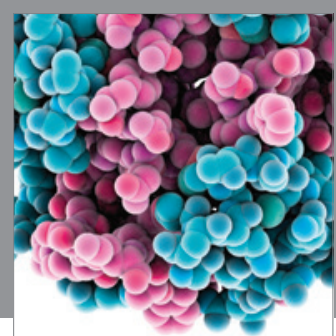

Journal of
Diabetes Research

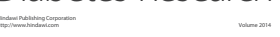

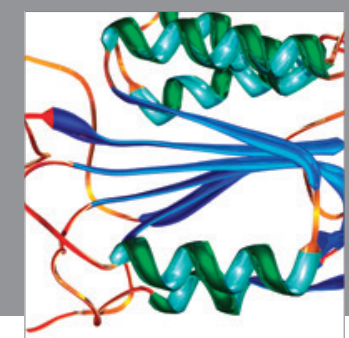

Disease Markers
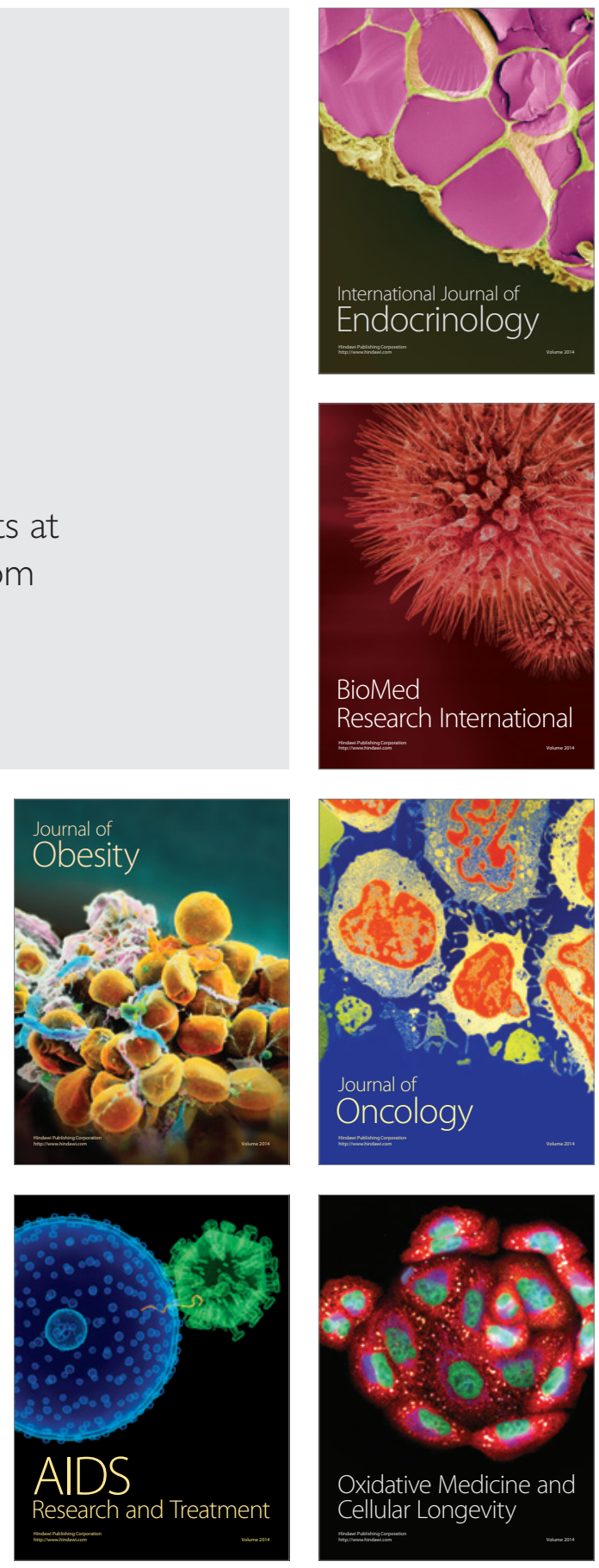